\title{
Identification methods and test results for tilting pad and fixed geometry journal bearing dynamic coefficients - A review
}

\author{
T.W. Dimond*, P.N. Sheth, P.E. Allaire and M. He \\ Rotating Machinery and Controls (ROMAC) Lab, Mechanical and Aerospace Engineering, University of Virginia, \\ Charlottesville, VA 22901, USA
}

\section{Received 15 August 2007}

Revised 5 December 2007

\begin{abstract}
Fluid film journal bearings (FFBs) are used to support high-speed rotors in turbomachinery which often operate above the rotor first bending critical speed. The FFBs provide both lateral support and dynamic coefficients: stiffness, damping, and mass terms, related to machine vibrations. Detailed numerical values of the bearing dynamic characteristics are necessary for proper design and operation of rotating machinery.

The methods of the identification of fluid film journal bearing static and dynamic characteristics, particularly the bearing stiffness, damping, and mass coefficients, from measured data, obtained from different measurement systems, is reviewed. Many bearing tests have been performed to validate a number of different theoretical models, including the classical Reynolds isoviscous model. More advanced bearing models include the thermohydrodynamic (THD), and thermoelastohydrodynamic (TEHD) approaches. The advanced models also include turbulence effects which are important as rotor speeds continue to increase. The range of measured bearing data no longer includes current operational conditions.

The various approaches to the bearing identification problem are discussed, including the different force excitation methods of incremental loading, sinusoidal, pseudorandom, impulse, known/additional unbalance, and non-contact excitation. Also bearing excitation and rotor excitation approaches are discussed. Data processing methods in the time and frequency domains are presented. Methods of evaluating the effects of measurement uncertainty on overall bearing coefficient confidence levels are reviewed.

In this review, the relative strengths and weaknesses of bearing identification methods are presented, and developments and trends in improving bearing measurements are documented. Future trends in journal bearing identification improvement are discussed.
\end{abstract}

Keywords: Fluid film bearing identification, stiffness, damping, inertia, uncertainty

\section{Introduction}

Fluid film journal bearings provide lateral rotor support. They are also the source of bearing stiffness, damping, and mass properties in rotor bearing systems. The proper design of these bearings is required for the successful industrial operation of rotating machinery. As machine speeds and loads and instability drivers increase, there is an increasing need for more accurate fluid film bearing identification carried out for the high surface speeds of today's machines. Several bearing modeling issues, such as whether synchronous vs. non-synchronous tilting pad bearing coefficients are used, which turbulence model is used, which thermal heating model is used, which lubricant

\footnotetext{
*Corresponding author. Tel.: +1 434243 4934; Fax: +1 434982 2246; E-mail: twd5c@virginia.edu.
} 
starvation model is used, have major importance in rotor dynamic system analysis. Additional issues include the current emphasis on reduced oil flow and new pad materials in tilting pad bearings to reduce temperature rise and the effects of turbulence with journal surface speeds in the range of $150 \mathrm{~m} / \mathrm{s}$.

The support forces and dynamic properties in fluid film bearings arise from the fluid-structure interaction forces between the rotor and the bearing fluid film. These properties were originally described by Reynolds using a laminar, isoviscous fluid model. More advanced, modern bearing theories have been developed, including THD and TEHD bearing models which describe bearing hydrodynamics with significant heating of the lubricant and elastic deformations of the bearing solid components [50]. Lubricant starvation effects and turbulence must be accurately modeled [50]. The models with increased complexity are required to provide more accurate theoretical bearing parameters, as test data is quite limited. The stiffness, damping, and mass coefficients arise from a perturbation solution to the elastohydrodynamic and thermal equations of the model used. However, there is model sensitivity to design parameters such as bearing clearances, changes in viscosity, manufacturing tolerances, thermal growth, and elastic deformation. The experimental validation of the bearing models is vital.

Generally, two distinct approaches to exciting the rotor-bearing system for dynamic coefficient identification have been used. One approach, that resembles real machine operation, involves holding the bearing housing rigidly while exciting a moving shaft. The other approach, referred in this work as the inverse method, holds the shaft rigidly while exciting the moving bearing housing. For lubricant flows in fluid film bearings, the approaches are dynamically equivalent and either is valid in measuring bearing coefficients when performed properly.

Most experimenters have performed dynamic coefficient identification with a rotor that is dynamically rigid with no bending critical speeds in the operating region, although some have considered a flexible rotor-bearing system. A few have also considered the effects of compliant foundations in the total rotor-bearing system.

With both the regular and inverse approaches, various excitation signal forms have been applied, including sinusoidal, transient, known/additional unbalance, and pseudorandom. A new trend has emerged with the availability of non-contact excitation such as through magnetic bearings. Both sinusoidal and pseudorandom approaches can be used for applying forces to the rotor-bearing system using active magnetic bearings or non-contact electromagnetic shakers as force exciters. Many of the older published reports describing bearing identification experiments have not presented confidence intervals for the measured coefficients, but more recent experimenters have included them in their published work $[15,29]$.

\section{Dynamic equations of motion and bearing coefficients}

The equations of motion for fixed pad bearings are relatively well known and not repeated here [60]. Also, the industrial use of fixed pad bearings in advanced machines has been declining for many years due to instability problems in high speed flexible rotors resulting in their widespread replacement by tilting pad bearings. The full equations of motion for a tilting pad rigid-rotor bearing system are [50]:

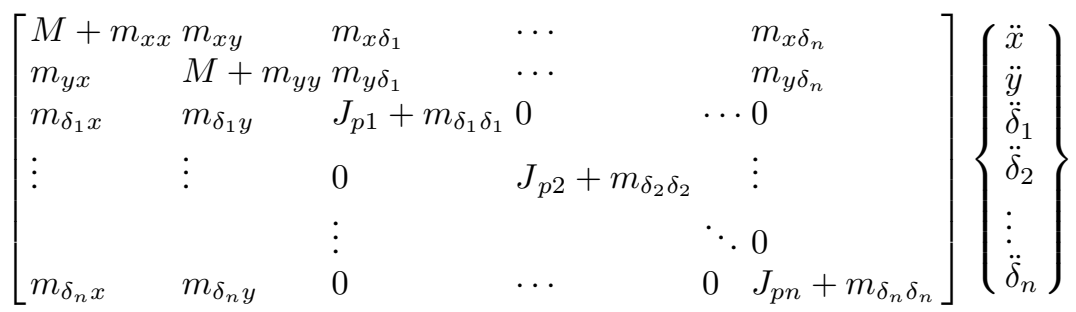

$$
\begin{aligned}
& +\left[\begin{array}{llllll}
c_{x x} & c_{x y} & c_{x \delta_{1}} & \cdots & & c_{x \delta_{n}} \\
c_{y x} & c_{y y} & c_{y \delta_{1}} & \cdots & & c_{y \delta_{n}} \\
c_{\delta_{1} x} & c_{\delta_{1} y} & c_{\delta_{1} \delta_{1}} & 0 & \cdots & 0 \\
\vdots & \vdots & 0 & c_{\delta_{2} \delta_{2}} & \ddots \\
& & \vdots & \ddots & \ddots & 0 \\
c_{\delta_{n} x} & c_{\delta_{n} y} & 0 & \cdots & 0 & c_{\delta_{n} \delta_{n}}
\end{array}\right]\left\{\begin{array}{l}
\dot{x} \\
\dot{y} \\
\dot{\delta}_{1} \\
\dot{\delta}_{2} \\
\vdots \\
\dot{\delta}_{n}
\end{array}\right\}
\end{aligned}
$$




$$
+\left[\begin{array}{llllll}
k_{x x} & k_{x y} & k_{x \delta_{1}} & \ldots & & k_{x \delta_{n}} \\
k_{y x} & k_{y y} & k_{y \delta_{1}} & \cdots & & k_{y \delta_{n}} \\
k_{\delta_{1} x} & k_{\delta_{1} y} & k_{\delta_{1} \delta_{1}} & 0 & \cdots & 0 \\
\vdots & \vdots & 0 & k_{\delta_{2} \delta_{2}} & \ddots & \vdots \\
& \vdots & \ddots & \ddots & 0 \\
k_{\delta_{n} x} & k_{\delta_{n} y} & 0 & \cdots & 0 & k_{\delta_{n} \delta_{n}}
\end{array}\right]\left\{\begin{array}{l}
x \\
y \\
\delta_{1} \\
\delta_{2} \\
\vdots \\
\delta_{n}
\end{array}\right\}=\left\{\begin{array}{l}
F_{x} \\
F_{y} \\
0 \\
0 \\
\vdots \\
0
\end{array}\right\}
$$

The terms are defined in the nomenclature. The $x$ and $y$ terms and their derivatives refer to lateral displacements, velocities, and accelerations in the horizontal and vertical directions, respectively. The $\delta$ terms and their derivatives refer to the rotational displacements, velocities, and accelerations of the individual pads about the pivots.

There are three terms on the left hand side of Eq. (1): mass, damping and stiffness matrices in that order. In the mass matrices, the mass terms denoted by $\mathrm{M}$ indicate the mechanical shaft mass while the terms $J_{i}$ designate mechanical pad angular mass moments of inertia. The mass terms denoted $m_{i j}, m_{i \delta_{j}}, m_{\delta_{i} j}, m_{\delta_{i} \delta_{j}}$ represent fluid inertia (added mass) effects in the lubricant film. For laminar flow bearings with small clearance ratios and reduced Reynolds number, these fluid added mass terms are zero. In the damping and stiffness matrices, the terms denoted as $c_{i j}, k_{i j}$ are the principal and cross coupled damping and stiffness value while the $c_{i \delta_{j}}, c_{\delta_{i} j}, c_{\delta_{i} \delta_{i}}, k_{i \delta_{j}}, k_{\delta_{i} j}, k_{\delta_{i} \delta_{i}}$ are the damping and stiffness terms related to the angular displacements and angular velocities of the pad.

Often the full non-synchronous equations for tilting pad bearings, as given above, are not used in rotordynamic analyses, although recent work shows the necessity of considering the full equations in stability analyses [12]. If the full frequency-dependent bearing coefficients given in Eq. (1) are reduced at a synchronous or non-synchronous excitation frequency, then the rigid rotor equations of motion reduce to the form

$$
\left[\begin{array}{ll}
M+m_{x x} & m_{x y} \\
m_{y x} & M+m_{y y}
\end{array}\right]\left\{\begin{array}{l}
\ddot{x} \\
\ddot{y}
\end{array}\right\}+\left[\begin{array}{ll}
c_{x x} & c_{x y} \\
c_{y x} & c_{y y}
\end{array}\right]\left\{\begin{array}{l}
\dot{x} \\
\dot{y}
\end{array}\right\}+\left[\begin{array}{ll}
k_{x x} & k_{x y} \\
k_{y x} & k_{y y}
\end{array}\right]\left\{\begin{array}{l}
x \\
y
\end{array}\right\}=\left\{\begin{array}{l}
F_{x} \\
F_{y}
\end{array}\right\}
$$

where the pad degrees of freedom are assumed to have the reduction frequency $\Omega$. When the full coefficients from Eq. (1) are reduced to the form in Eq. (2), typically the reduction is accomplished synchronously [4].

Equation (2) is also appropriate for fixed-geometry bearings where the dynamic coefficients are excitationfrequency independent at a given running speed. For bearings where the lubricant flow is laminar and the clearance ratio is small, the above inertia coefficients denoted by $m_{i j}$ are taken to be zero. However, it has been demonstrated that the inertia coefficients are important for near-turbulent bearings when the pad Reynolds number, defined as

$$
R e=\frac{R \omega c_{p}}{\nu}
$$

is on the order of $10^{2}$ [24].

Equation (1) can also be expressed in matrix form:

$$
\mathbf{M u ̈}+\mathbf{C u}+\mathbf{K u}=\mathbf{f}
$$

If a flexible rotor or compliant foundation is considered, the order of Eq. (4) can be expanded to account for the additional degrees of freedom. Many bearing identification experiments express this equation in the frequency domain and obtain experimental frequency response functions (FRFs) of the form

$$
\mathbf{H u}=\mathbf{f} ; \quad \mathbf{H}=-\Omega^{2} \mathbf{M}+j \Omega \mathbf{C}+\mathbf{K}
$$

Or equivalently, Eq. (5) is expressed as:

$$
\mathbf{u}=\mathbf{Z} \mathbf{f} ; \mathbf{H}=\mathbf{Z}^{-1}
$$

The elements of the FRF matrix can then be curve-fitted to extract the bearing coefficients.

Equations (4) and (5) can also be inverted so that the coefficients to be identified appear in a vector of unknowns, and the measured displacements/accelerations appear in a matrix. If Eq. (4) is used to determine Eq. (7), then the solution is found in the time domain; if Eq. (5) is used, the solution is found in the frequency domain.

$$
\mathbf{U} \beta=\mathbf{f}
$$

For tilting pad bearings, Eq. (7) is underconstrained for a single system perturbation, since there are more unknowns than available equations. Multiple perturbations are normally used in tilting pad bearing measurements to obtain an overconstrained problem, which is then solved using least squares [14,67]. 


\section{Previous literature reviews}

Someya compiled an extensive listing of experimental data which is compared with analytical results for hydrodynamic journal bearings, including plain journals with axial oil grooves, two-lobe bearings, and tilting pad bearings [76]. In several cases, he noted that there were large differences between theory and experiment, but the reported theoretical results used for the comparisons by Someya were calculated using an isoviscous lubricant assumption [76]. This limitation on the theoretical results was noted, but some of the differences were attributed to non-linearities in the oil film. Although some of the reported data exhibited considerable scatter, there was no published uncertainty analysis or confidence intervals provided for the identified bearing parameters in the paper. Other reported data showed little scatter, depending upon the bearing tested. Some coefficients showed as little as approximately 5 percent differences between predicted and identified values, with a typical approximate difference of 20 percent. When scatter was significant, differences of 100-200 percent were found.

Goodwin reviewed the experimental techniques used in measuring bearing coefficients [51]. The review considered static loading, shakers, unbalance forcing, and transient methods. Comparisons of the relative complexities of the methods were presented. He found that the typical agreement of the various experimental methods with theoretical values was within 20 percent, though some cases of discrepancies of 100 percent or more had been reported by some authors. Swanson and Kirk provided a literature review of fixed-geometry bearing tests in tabular format [25].

An extensive survey of a wide variety of bearing and seal identification tests for dynamic coefficients was recently presented by Tiwari [66]. This survey included fixed-geometry and tilting pad journal bearings, rolling element bearings, thrust bearings, seals, active magnetic bearings, and other rotordynamic components.

This literature review is more limited in scope than the Tiwari review and concentrates on journal bearings, fixed and tilting pad [66]. The emphasis is on the experimental methods employed to identify tilting pad and fixed geometry journal bearing dynamic coefficients. This review discusses the specific methods that have been developed for bearing coefficient identification, discusses the results and notes improvements in these methods that addressed data quality. Improvements that address issues such as noise and scatter reduction and improvement of the confidence intervals are noted. This review also considers the current industrial trends in higher journal surface speeds which lead to increases in turbulent effects and inertia effects in the theoretical models. If sufficient data was presented to estimate a bearing Reynolds number, or if the Reynolds number is reported, then this is also presented.

\section{Experimental methods}

This section discusses the literature involving the various primary excitation methods employed in journal bearing identification. Detailed assessments of the results are presented.

\subsection{Incremental loading - Bearing stiffness}

An experiment that requires less equipment than the other approaches to conduct is the incremental loading of journal bearings to measure the lubricating film stiffness without considering damping or fluid mass data. The stiffness is measured by applying different static loads to the shaft or bearing housing and measuring the displacement. No external method of vibration is required. Experimenters utilizing incremental loading are summarized in Table 1.

Mitchell et al. [41] used this method to identify the stiffness coefficients for a plain journal bearing. A photograph of the test rig is shown in Fig. 1. Loads were incrementally applied to the bearing housing while holding the shaft in rigid bearings. The stiffness coefficients were found by measuring the influence coefficients from incremental horizontal and vertical forces:

$$
a_{x x}=\frac{x_{1}}{\Delta F_{x}} ; a_{y x}=\frac{y_{1}}{\Delta F_{x}} ; a_{y y}=\frac{y_{2}}{\Delta F_{y}} ; a_{x y}=\frac{x_{2}}{\Delta F_{y}}
$$

The influence coefficient matrix was then formed, and the stiffness coefficients were found by matrix inversion:

$$
\mathbf{a}=\left[\begin{array}{ll}
a_{x x} & a_{x y} \\
a_{y x} & a_{y y}
\end{array}\right] ; \quad \mathbf{K}=\mathbf{a}^{-1}
$$




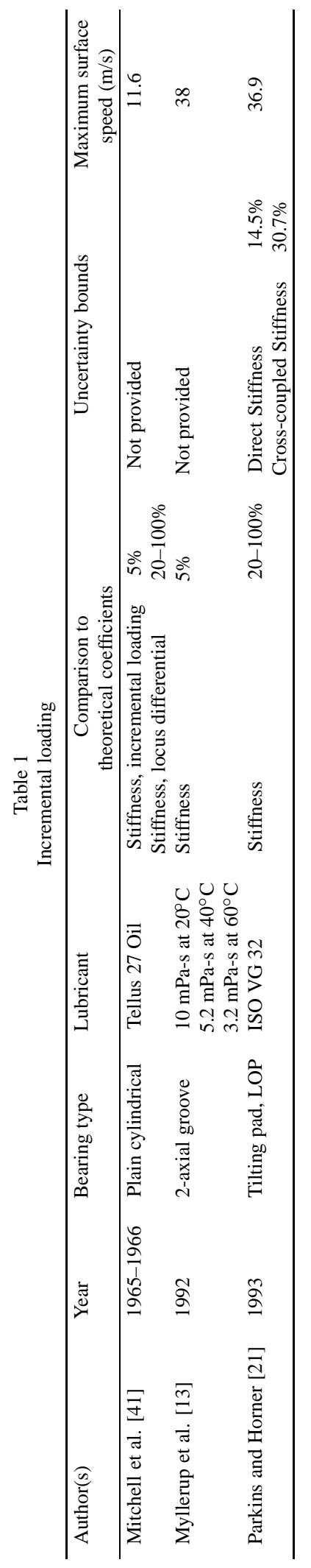




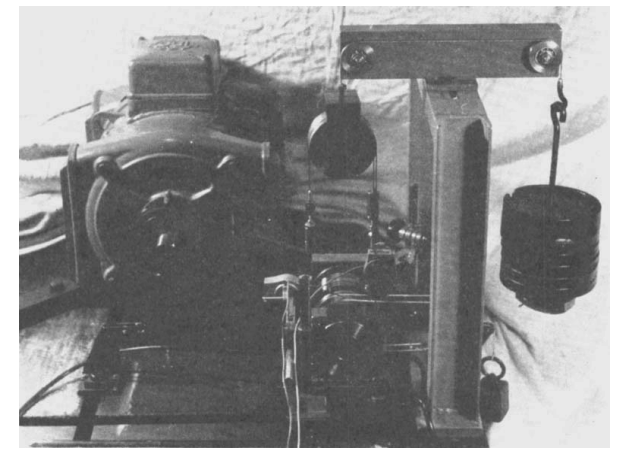

Fig. 1. Mitchell et al. [41]. Test rig for incremental static loading.

The influence coefficient method above was compared to the locus differentiation method, a semigraphical method that takes data from curves of attitude angle versus eccentricity and dimensionless force versus eccentricity. The data was applied to the following equations to calculate influence coefficients.

$$
\begin{aligned}
& \frac{c_{p}}{F} a_{x x}=\frac{1}{F} \frac{\partial F}{\partial \varepsilon} \cos \phi_{0} \\
& \frac{c_{p}}{F} a_{y y}=\frac{1}{\varepsilon} \cos \phi_{0}-\frac{\partial \phi_{0}}{\partial \varepsilon} \sin \phi_{0} \\
& \frac{c_{p}}{F} a_{x y}=\frac{1}{F} \frac{\partial F}{\partial \varepsilon} \sin \phi_{0} \\
& \frac{c_{p}}{F} a_{y x}=\frac{1}{\varepsilon} \sin \phi_{0}+\frac{\partial \phi_{0}}{\partial \varepsilon} \cos \phi_{0}
\end{aligned}
$$

Theoretical stiffness values were obtained from short bearing theory.

Myllerup et al. [13] measured the stiffness coefficients of a two-axial groove cylindrical bearing by the locus differentiation method. The potential for matrix ill-conditioning in inverting the influence coefficient matrix was cited as the rationale for using the locus differentiation method. As a result, locus differentiation was employed in terms of the stiffness coefficients of the form

$$
\begin{aligned}
k_{x x} & =\frac{d F}{d \varepsilon} \cos \phi_{0} \\
k_{x y} & =\frac{d F}{d \varepsilon} \sin \phi_{0} \\
k_{y x} & =-\frac{F}{\varepsilon} \frac{d}{d \varepsilon}\left(\varepsilon \sin \phi_{0}\right) \\
k_{y y} & =\frac{F}{\varepsilon} \frac{d}{d \varepsilon}\left(\varepsilon \cos \phi_{0}\right)
\end{aligned}
$$

Curve fitting was used to smooth the derivatives with respect to eccentricity. It was found that this method is only applicable to journal bearings with geometric axial symmetry. The method becomes an approximation when the bearing does not have axial symmetry.

Parkins and Horner [21] identified the stiffness of tilting pad bearings using an incremental loading method. The influence coefficients were measured based on incremental loads. Problems arose due to the large difference in magnitudes between horizontal and vertical film stiffness values for load-on-pad (LOP) configurations. To overcome this, incremental loads were applied at oblique angles to the horizontal and vertical. This approach necessitated limiting the incremental load so that the bearing coefficients were not significantly affected, i.e. moving from a LOP to a load-between pad (LBP) configuration, where the coefficients were closer to isotropic. The results were compared to a TEHD model. There was significant scatter in the data, but qualitative trends in stiffness compared favorably to the model.

\subsection{Sinusoidal excitation}

One of the most popular methods for experimentally measuring the frequency response functions of the rotor bearing system is to perturb the system using a sinusoidal force input. The advantage is that sinusoidal excitation can be applied at any excitation frequency up to the exciter limits, although some experiments have been limited to synchronous excitation $[33,44,45,79]$. The bearing housing or journal responses to the external force input are measured at each applied excitation frequency. The bearing responses are either cast as experimental frequency 
response functions, Eq. (5) or Eq. (6), or as a coefficient matrix, Eq. (7), to perform the bearing identification of dynamic coefficients.

By selective application of the two orthogonal forces, the shapes of the vibration orbits can be controlled. Circular orbits result in singular or near singular matrices in Eq. (5), Eq. (6), or Eq. (7) [29,67], which is a big problem. Elliptical orbits are normally selected to improve the numerical conditioning of the matrix inversion required in either Eq. (5), Eq. (6), or Eq. (7). Other researchers have produced straight-line orbits to avoid matrix inversion altogether.

\subsubsection{Sinusoidal excitation without comprehensive uncertainty analysis}

Most early experiments utilizing sinusoidal excitation did not include comprehensive uncertainty analysis as part of the reported results. The papers describing experimental results for these experiments are summarized in Table 2.

Morton [61] performed full-scale testing on sleeve bearings for large turbines. The frequency response function matrix, given in Eq. (6), was non-dimensionalized and used to perform the bearing coefficient identification. Experimental bearing coefficients were compared to theoretical coefficients calculated with an isoviscous bearing model. The effects of variable viscosity and bearing solid elastic deformation were considered, but including a correction for these effects did not account for the discrepancy in this work. The rotor was held in slave bearings, which were not identified in the paper. If these were also fluid film bearings, the additional contributions to stiffness and damping were not accounted for.

Diana et al. [28] identified the stiffness and damping coefficients for elliptical bearings using two single frequency harmonic excitations. Two sinusoidal forces at different excitation frequencies were applied, and the resulting horizontal and vertical force components in the oil film were measured. The orientation of the exciters and the method of applying external forces were not reported. Using the frequency domain approach, Eq. (7) was solved to identify the bearing coefficients. In the paper, the experimentally identified bearing coefficients were compared to an isoviscous hydrodynamic bearing model with turbulence corrections.

An extensive study by Parkins [18,19] adjusted the amplitude and phase of the applied synchronous forces to produce straight-line shaft orbits. Sinusoidal forces were applied to the test bearing housing with electromagnetic shakers. The rotor was held in fixed rolling element bearings. Two distinct, straight-line orbits in the horizontal and vertical directions were used. From the time domain bearing response data $x(t)$ and $y(t)$, the bearing coefficients were identified using the following relationships. For the vertical straight-line orbit,

$$
\begin{aligned}
& \dot{y}=0, y \neq 0 ; F_{x}=k_{x y} y, F_{y}=k_{y y} y \\
& y=0, \dot{y} \neq 0 ; F_{x}=c_{x y} \dot{y}, F_{y}=c_{y y} y
\end{aligned}
$$

And for the horizontal straight-line orbit,

$$
\begin{aligned}
& \dot{x}=0, x \neq 0 ; F_{x}=k_{x x} x, F_{y}=k_{y x} x \\
& x=0, \dot{x} \neq 0 ; F_{x}=c_{x x} \dot{x}, F_{y}=c_{y x} \dot{x}
\end{aligned}
$$

The stiffness coefficients were also independently measured using the incremental loading method and application of Eq. (9). The methods did not require the measurement of the phase angle in shaft orbits, which was cited as a large source of error in other authors identified bearing coefficients. A first-order non-linear approximation to the bearing coefficients was also considered. As an example, the horizontal direct stiffness relation is

$$
k_{x x}=k_{x x 0}+k_{x x} x
$$

Similar equations to Eq. (14) were applied for the remaining stiffness and damping coefficients. The experimental bearing coefficients were compared to theoretical coefficients from a THD bearing model with an assumed temperature profile. The identified coefficients were found to be susceptible to noise and scatter when locating the zero crossings. This source of error could have been mitigated by using multiple replications and curve fitting but the use of this type of curve fitting was not discussed in the paper. The non-linear effects were not significant for eccentricity ratios below 0.78 .

Kanki et al. [33] investigated water-lubricated pump bearings using sinusoidal excitation. Since water was the lubricant, with low viscosity compared to oil, fluid inertia effects were important. This resulted in significant added mass coefficients. The rotor was run below the first critical speed and was supported in two cylindrical test bearings 


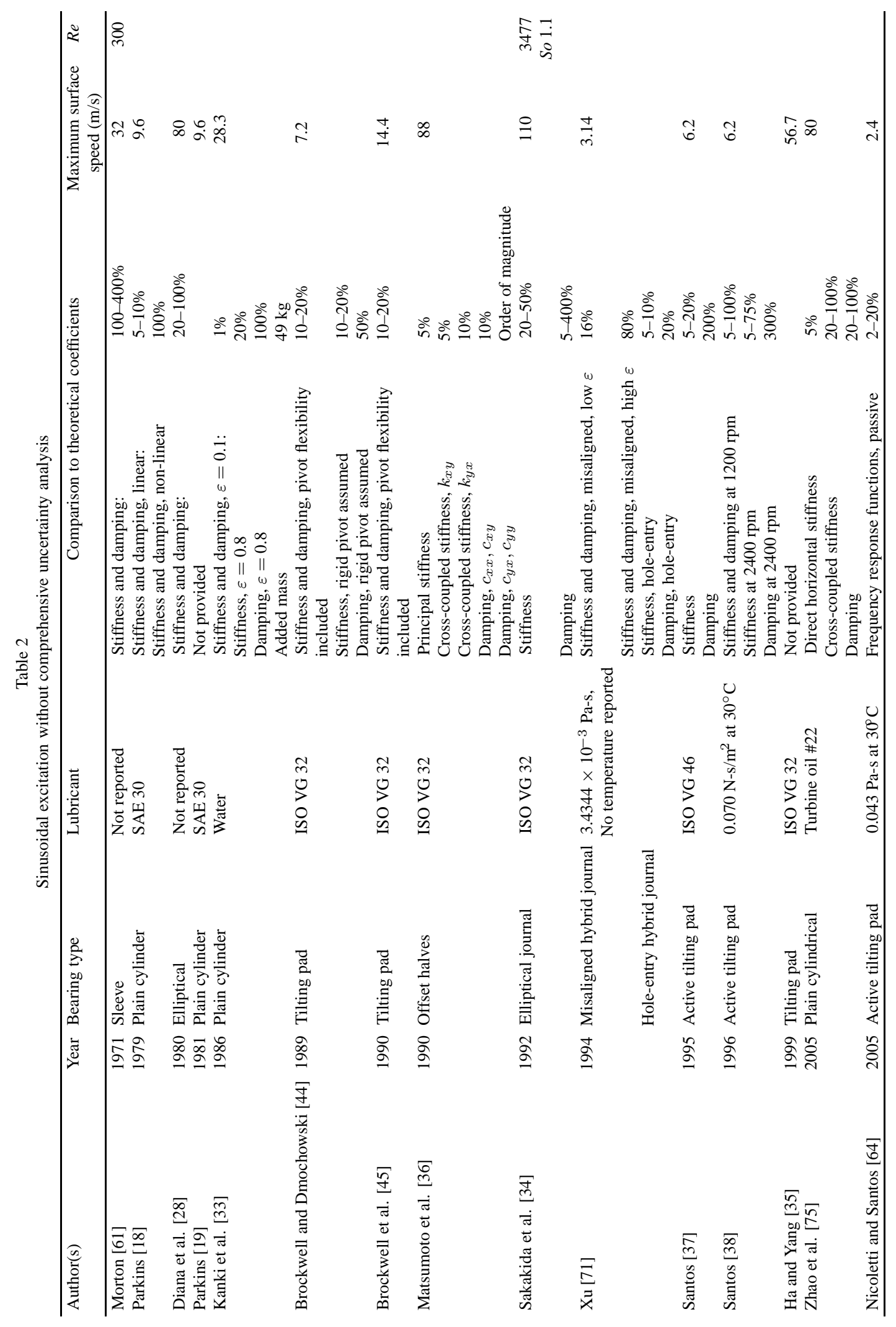




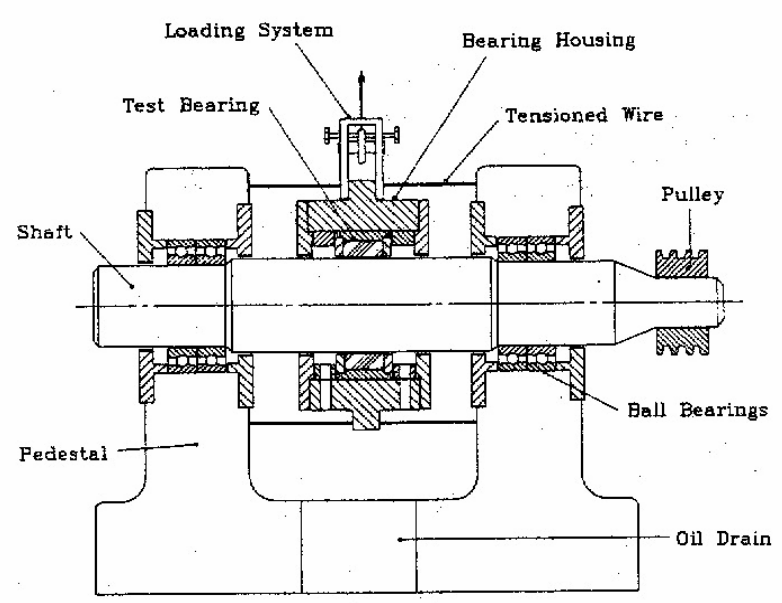

Fig. 2. Brockwell and Dmochowski [44] test rig.



Fig. 3. Equivalent system and bearing with shaft, after Brockwell and Dmochowski [44].

and two tilting pad bearings. The dynamic characteristics of the tilting pad bearings were not discussed. Static loads were applied to the test bearing housing with air bellows, and dynamic loads were applied to the housing using hydraulic exciters.

Two methods of applying the sinusoidal excitation were used. The exciters were used to produce forward and backward whirl at a single excitation frequency, and to produce forward whirl at two different excitation frequencies. Results from the two excitation methods were compared and found to be within 10 percent of each other, although the method using forward whirl and distinct excitation frequencies yielded results closer to theoretically predicted values. Significant added mass terms of $49 \mathrm{~kg}$ due to fluid inertia effects were observed.

The synchronous excitation selective vibration orbit method was also used by Brockwell and Dmochowski to identify tilting pad linear bearing coefficients [44]. The shaft was held fixed in rolling element bearings, and electromagnetic shakers were used to apply forces to the test bearing housing. A schematic of the test arrangement is shown as Fig. 2. Only synchronous excitations were applied to the bearing housing. The selective orbit method was further improved with damping coefficient corrections for shaft stiffness in bending and for pivot flexibility. The equivalent mechanical system is shown in Fig. 3. The direct bearing coefficients were found, from Eq. (13), as

$$
\begin{aligned}
k_{i i} & =\frac{k_{e q} k_{s}\left[k_{s}-\left(1-A_{d}\right) k_{e q}\right]-\omega^{2}\left(1-A_{d}\right) k_{s} c_{e q}^{2}}{\left[k_{s}-\left(1-A_{d}\right) k_{e q}\right]^{2}+\omega^{2}\left(1-A_{d}\right)^{2} c_{e q}^{2}} \\
c_{i i} & =\frac{k_{s}^{2} c_{e q}}{\left[k_{s}-\left(1-A_{d}\right) k_{e q}\right]^{2}+\omega^{2}\left(1-A_{d}\right)^{2} c_{e q}^{2}}
\end{aligned}
$$

where $A_{d}$ is the ratio of shaft deflection at the transducers to shaft deflection at the bearing mid span. It was found that shaft and pivot flexibility did not significantly affect the identified bearing direct stiffness values. Results were 
compared to a TEHD bearing model. The same scatter issues in zero crossings encountered by Parkins [18,19] were not addressed.

Brockwell et al. [45] reported additional results for the same rig, bearing and identification method for rotational speeds of $900 \mathrm{rpm}, 1800 \mathrm{rpm}, 2700 \mathrm{rpm}$, and $3600 \mathrm{rpm}$. Horizontal stiffness and damping values at $3600 \mathrm{rpm}$ were not reported due to a resonance in the test rig frame. The TEHD bearing model used to predict dynamic bearing coefficients, including a pivot flexibility correction, was also presented. The bearing model assumed that the journal and pad motion was synchronous with running speed. Bearing damping was over predicted by the model by up to 20 percent, as compared to experiment.

Matsumoto et al. [36] identified bearing stiffness and damping characteristics for an offset-halves journal bearing. Excitation forces were applied to the test bearing housing, and the shaft was held in rolling element bearings. The exact method for coefficient identification is not described, but QR decomposition was used to calculate the damping ratio. The experimental results were compared to a THD model of the offset-halves bearing. The scatter in the experimental damping coefficients was more pronounced than the scatter in experimental stiffness coefficients. The THD model was then applied to various bearing configurations for a comparison of stability. A four-pad tilting pad bearing gave the best stability margin, with an offset bearing with offset ratio of 0.75 found to be the next best option.

Sakakida et al. [34] identified the dynamic bearing coefficients for a $584.2 \mathrm{~mm}$ elliptical journal bearing. The test rotor was held in $406.4 \mathrm{~mm}$ elliptical journal bearings. The rigidity of the rotor and support bearings was claimed to be "sufficiently high", but the stiffness and damping of the support journal bearings was not quantified. The coefficients were identified by measuring forces, displacements, and accelerations for two independent shaft orbits and solving Eq. (5) in a least-squares sense. The rotor orbit was kept to a maximum value of approximately 10 percent of the clearance circle to obtain a suitable signal-to-noise ratio while not violating the linearity assumption. There were significant differences in cross-coupled stiffness and vertical damping, though qualitative trends matched predictions. Differences were attributed to unmodeled turbulence, fluid inertia, and bearing metal thermal effects.

$\mathrm{Xu}$ [71] identified bearing stiffness and damping coefficients for misaligned hybrid journal bearings. The test shaft was supported in high-stiffness hydrostatic bearings, with the loaded test bearing in the middle of the shaft. The stiffness of the hydrostatic slave bearings was not quantified. A rigid rotor was used to perform tests. Dynamic loading was applied to the test bearing housing with electromagnetic shakers. Forces and displacements were measured, and bearing coefficients were identified in the frequency domain using a least squares solution to Eq. (7). Experimental results were compared to an isoviscous hydrodynamic bearing model. The stiffness of the hydrostatic support bearings was not quantified. Un-modeled effects, including variable viscosity, were acknowledged as an influence on results, especially at high eccentricity ratios. Differences in load capacity and attitude angle at high eccentricity for the recessed hydrostatic bearing were attributed to manufacturing differences compared to the ideal bearing model. However, the high eccentricity case also resulted in an increase in lubricant temperature, so the isoviscous lubricant assumption was violated. The experimental results for principal horizontal stiffness and damping in the tested hybrid bearing were compared to theoretical values for the hybrid bearing, the tested hydrodynamic bearing, and the tested hydrostatic bearing. The model consistently overpredicted the bearing stiffness and damping values.

Zhao et al. [75] identified bearing coefficients using a test rig with a rigid rotor and non-synchronous sinusoidal force input capability. The tested plain journal bearing housing was allowed to move relative to the test shaft. However, the shaft was also supported in two five-pad tilting pad bearings. Static loading was applied using air bellows, and the test bearing was constrained from axial motion and axial misalignment using rollers against the axial faces of the test bearing. The only constraint on bearing radial motion other than oil film stiffness and damping was roller friction. Excitation forces were measured using pressure sensors. Displacements were measured using eddy-current probes, while velocities and accelerations were calculated using numerical differentiation with low-pass filtering. The measured and calculated displacement, velocity, and acceleration data were then used to identify bearing coefficients in the time domain using a least squares solution to Eq. (7). Experimental results were compared to theoretical coefficients calculated from a thermohydrodynamic analysis. Scatter in the data was attributed to nonlinearities in the oil film, and to noise in the calculated velocity. The noise effects were more pronounced in the estimation of damping parameters. The flexibility introduced by the tilting-pad support bearings was not included in the analysis, which would strongly influence the identified direct stiffness and damping terms. The uncertainty in identified coefficients, which would influence the conclusion of nonlinear oil film behavior based on scatter, was not addressed. 
Ha and Yang [35] performed non-synchronous excitation of a five-pad tilting pad journal bearing, LOP, to investigate the frequency dependence of the dynamic coefficients. The bearing was loaded statically with an air bellows. Dynamic excitation was performed sinusoidally on the bearing casing with hydraulic exciters. The shaft was held rigidly in rolling element bearings. Excitation frequencies ranged from 0.6 to 0.9 times the rotational speed. The shaft and bearing motion were measured using displacement sensors and piezoelectric accelerometers. Equation 7 was used for identification in the frequency domain. The identified stiffness and damping were weakly dependent on excitation frequency. However, any possible frequency dependence could not be evaluated due to the lack of an uncertainty analysis.

Santos $[37,38]$ applied active control to tilting pad bearing pivots. Identification of the bearing dynamic coefficients was required for feedback to the controller. The dynamic coefficients were controlled by adjusting the clearances between the pad and pivot via a flexible membrane on the bearing pivot. The flexible membrane was actuated by hydraulic pressure. Static and dynamic loads were applied to the shaft with an attached lever. The lever was dynamically excited with a piezoelectric actuator, which applied dynamic loads to the shaft. The lever restricted horizontal movements, so only vertical coefficients were identified. Identification was performed non-synchronously using a form of Eq. (7) that incorporated the additional degrees of freedom introduced by the lever and actuator. The low frequency stiffness was identified by exciting the lever at $5 \mathrm{~Hz}$ at shaft rotational speeds of 1200 and $2400 \mathrm{rpm}$. The theoretical and identified stiffness and damping were compared for excitation frequencies from $0-200 \mathrm{~Hz}$ at a rotational speed of $2400 \mathrm{rpm}$. The theoretical damping was higher than the identified damping. The rotorlever-bearing transfer function were also identified for coefficients were identified for different flexible membrane actuation pressures. Experimentally identified bearing coefficients were compared to a Reynold's equation solution using Chebyshev polynomials to represent the pressure profile. It was not clear from the paper whether an isoviscous lubricant was assumed

Nicoletti and Santos [64] also investigated load-on-pad active tilting pad bearings. The bearing dynamic coefficients were controlled by injecting oil through the pads. Dynamic forces were applied with an electromagnetic shaker attached to an excitation rolling element bearing on the shaft. Forces were measured with a load cell attached to the shaker. The shaft displacement was measured with eddy current probes. Identification was accomplished with Eq. (7). The cross-coupled bearing coefficients were considered negligible and were not included in the identification procedure.

\subsubsection{Sinusoidal excitation with comprehensive uncertainty analysis}

More recent experiments utilizing sinusoidal excitation include a comprehensive uncertainty analysis as part of the reported results. However, some of the earliest experiments utilizing sinusoidal excitation did attempt to quantify uncertainty. The papers describing experimental results for these experiments are summarized in Table 3.

Glienicke [40] excited turbine bearings using synchronous sinusoidal excitation. The exciters were phase displaced by $\pi / 2$, so they could be set at the same amplitude and simulate an additional unbalance excitation. Identification was performed in the frequency domain by measuring displacement amplitudes and phase angles resulting from input forces. However, the details of the calculation were not provided.

Hisa et al. [70] identified bearing stiffness and damping coefficients for a $508 \mathrm{~mm}$ tilting-pad bearing and an $812.8 \mathrm{~mm}$ elliptical bearing. Loading applied orthogonally loading to the individual bearing housings was used to generate two linearly independent shaft orbits. Input forces and output displacements of the bearing housing were measured, and Eq. (5) was then solved to identify the bearing coefficients. The rotor behaved rigidly over the range of operating speed and excitation, with a reported natural frequency of $130 \mathrm{~Hz}$. The operating range was 500-3600 rpm for the $508 \mathrm{~mm}$ bearing and $1200-1800 \mathrm{rpm}$ for the $812.8 \mathrm{~mm}$ bearing. The maximum excitation frequency was $60 \mathrm{~Hz}$. Vibration at the support bearings was measured so that the effects of the support bearings on test bearing measurements could be removed. The vibration magnitude and bearing coefficients at the support bearings were not reported. The identified bearing coefficients were compared to theoretical values calculated using an isoviscous bearing lubricant model. The lubricating oil exit temperature was used to determine the representative oil viscosity. The effect that the individual measurement errors had on overall uncertainty was discussed, but a systematic overall uncertainty analysis was not applied.

Parkins [20] extended the selected orbit method to allow damping measurements from a single, figure- 8 orbit. Coordinate transformations from a non-orthogonal, orbit aligned coordinate system with origin at the cross-over 


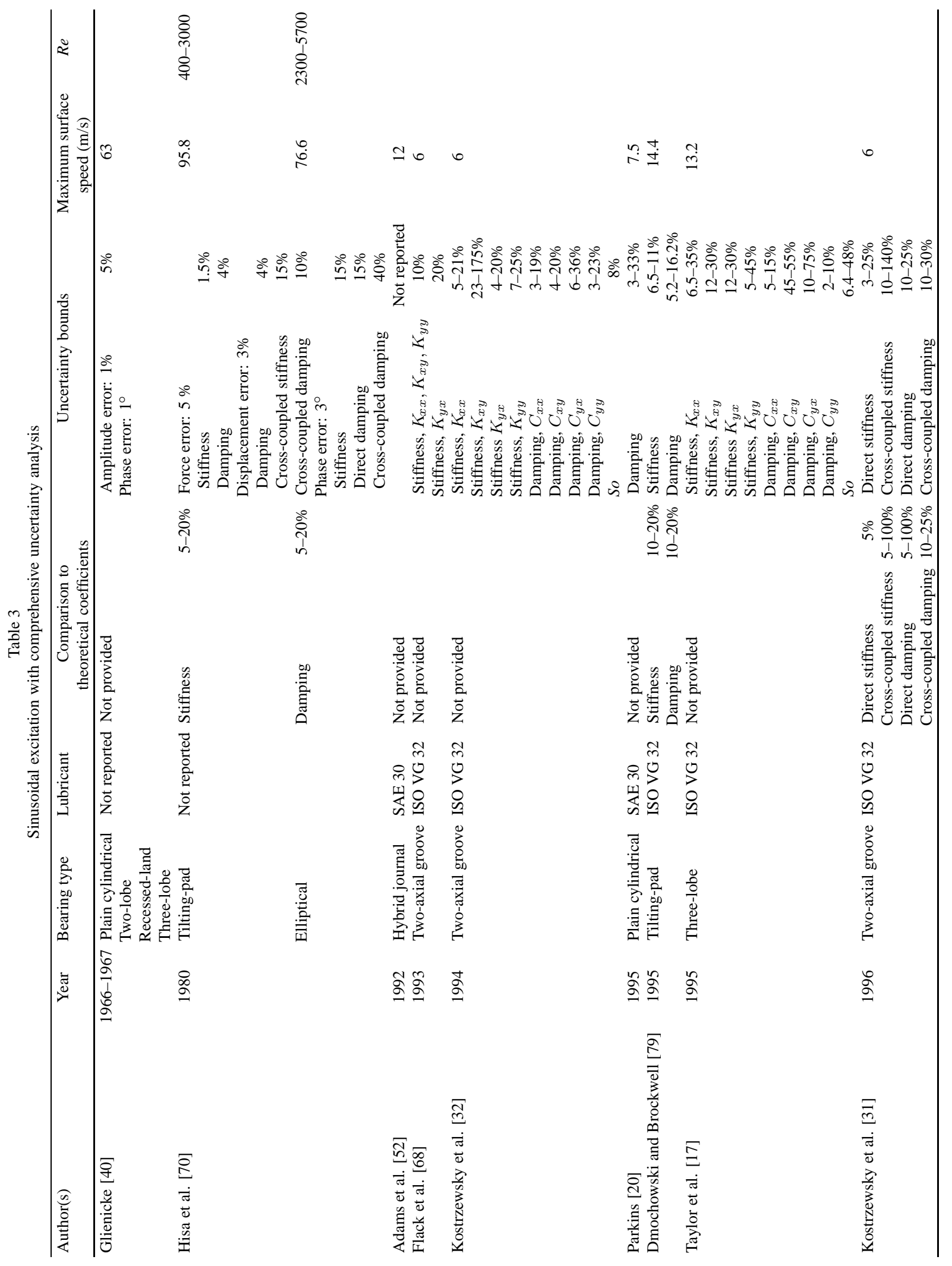




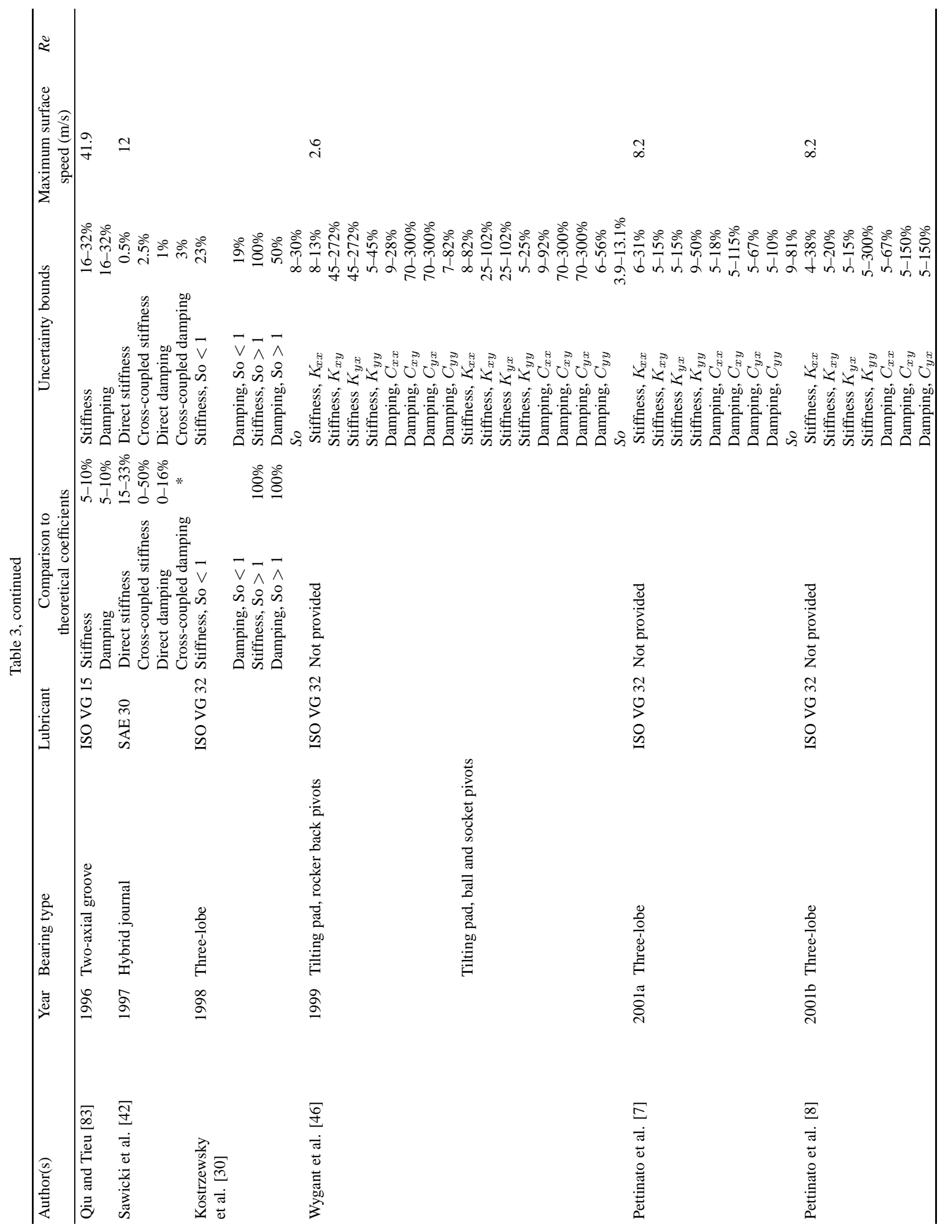




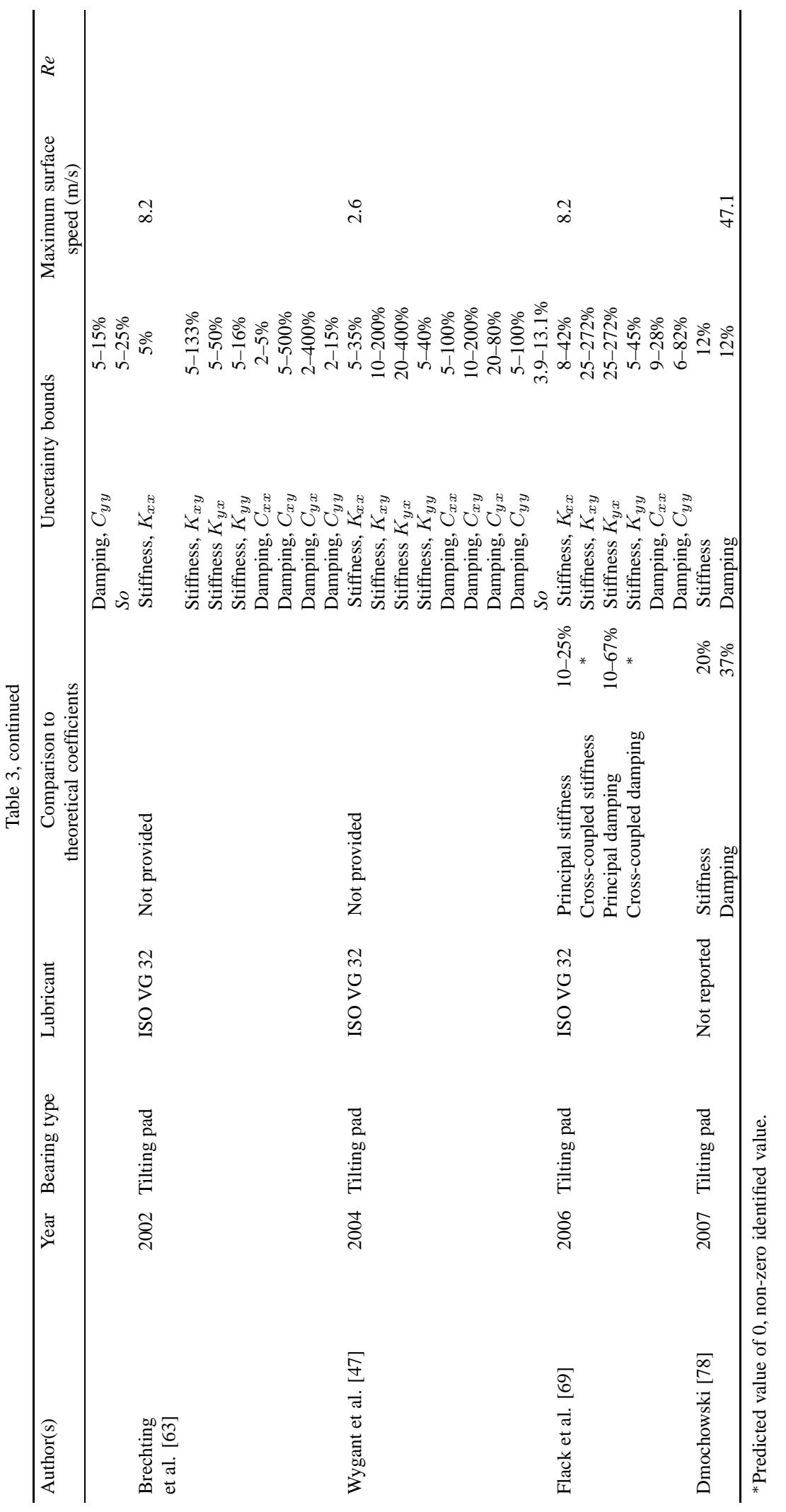




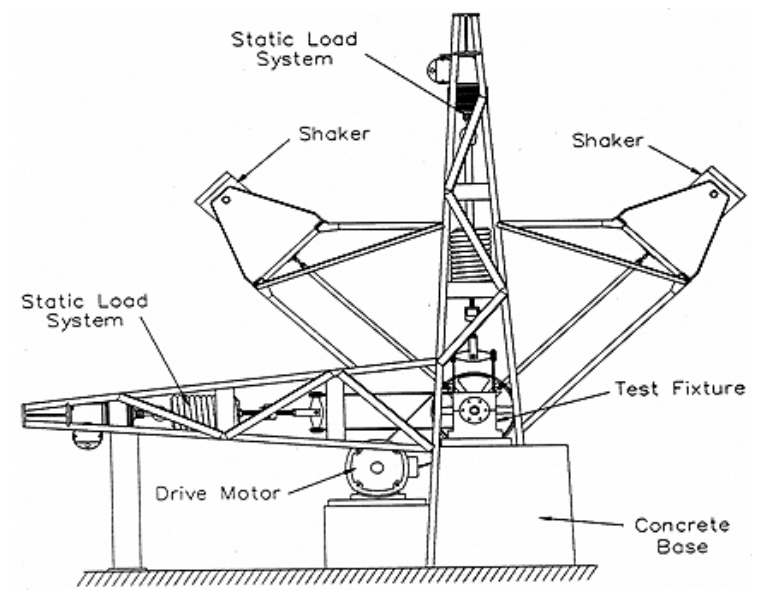

Fig. 4. Flack et al. [68] test rig.

point were used. The selected orbit method was further improved by the estimation of the effect of coordinate transformations, zero-force assumptions, and curve fitting on measurement uncertainty. Measurement uncertainty was most sensitive to the angle between the $p$ and $q$ axes; near orthogonality resulted in the lowest uncertainty.

Dmochowski and Brockwell [79] used the same rig and test method [44] to identify bearing coefficients for five pad tilting pad bearings in a LBP configuration with varying preloads. The test method was improved from their previous work by including an uncertainty analysis of identified bearing coefficients similar to that developed by Kostrzewsky and Flack [29]. The findings indicated that increased preload did not significantly affect bearing stiffness, but decreased the vertical damping by 60 percent at slower shaft speeds. For a given bearing load, an increase in $\mathrm{L} / \mathrm{D}$ ratio resulted in a reduction of bearing stiffness. This was because the bearing unit load was decreased due to the increase in L/D ratio. However, this change did not have a significant effect on the damping terms.

A rigid rotor test rig with non-synchronous sinusoidal excitation capabilities was described by Flack et al. [68]. Figure 4 gives a schematic of the test rig. Dynamic forces were applied to the bearing housing, and the shaft was held in rolling element bearings. Orbit magnitude and phase data were averaged, and bearing coefficients were estimated using Eq. (7). Row and column operations were performed on Eq. (7) to make it block-diagonal, which improved the numerical conditioning of the $\mathbf{U}$ matrix inversion. A comprehensive uncertainty analysis for the test rig was developed, based on single-sample uncertainty estimation [29,74]. The uncertainty analysis did not directly address loss of numerical precision due to matrix inversion, which was part of the bearing identification algorithm. The optimal points in shaft orbits to extract the bearing parameters were addressed, which are related to numerical conditioning. The uncertainty analysis was used on subsequent bearing identification experiments, including 2-axial groove bearings [31,32], 3 lobe bearings [7,8,17,30], and five-pad tilting pad bearings [46,47,63, 69]. Typical uncertainties in identified bearing coefficients were 3-20 percent, depending on the experiment. Over the life of the rig, surface speeds ranged from $2.6 \mathrm{~m} / \mathrm{s}-28.7 \mathrm{~m} / \mathrm{s}$.

The stiffness and damping results for 2-axial groove bearings were based on synchronous and non-synchronous force input. Typical average uncertainties were 12 percent for coefficients and 8 percent for So [32]. The tests indicated that dimensionless stiffness and damping coefficients are solely a function of $S o$ for journal bearings with laminar lubricant behavior.

Comparisons were also presented for predicted coefficients based on a finite element THD analysis [31]. The THD analysis incorporated an assumed axial pressure profile, variable viscosity, and a linearized turbulence model. The coefficients were identified from two independent orbits. Experimental horizontal direct damping was higher than predicted horizontal direct damping. The qualitative trend in cross-coupled damping was identified, but calculated cross-coupled damping fell outside the confidence interval of the experimentally identified cross-coupled damping.

The experimental methods were improved by selecting orbits that improved the numerical condition of the matrix inversion required for the identification method. Three independent shaft orbits were produced, and the orbits resulting in the lowest uncertainty were used to identify bearing coefficients for a 3-lobe journal bearing with 0.75 
preload $[17,30]$. Other studies of the 3-lobe bearing include testing of the linearity assumption the effect of load orientation on coefficients $[7,8]$.

Tests on tilting pad journal bearings included investigating the effect of load angle on the identified bearing coefficients [63]. Confidence intervals for the stiffness and damping coefficients were reported, but confidence intervals in the experimental So were not reported. The stiffness and damping coefficients were identified as a function of load angle, and it was found that the stability margin was not adversely affected within the confidence in the measurements.

Other five-pad tilting pad tests have included the effect of pad pivot friction [46] and varying preload [47] on identified bearing coefficients. Synchronous sinusoidal dynamic forces were applied. Surface speeds were limited to $2.6 \mathrm{~m} / \mathrm{s}$ in an effort to minimize temperature and turbulence effects. Cross-coupled stiffness coefficients were identified for sliding-friction pivots. The sliding friction pivot effect was more pronounced for lightly loaded bearings, indicating that the moments tending to align the pad to the rotor could not overcome the friction force.

The effect of non-synchronous excitation of tilting pad bearings were also investigated [69]. Results were compared to a TEHD model. Excitation frequencies of $0.5 \mathrm{X}, 1 \mathrm{X}$, and $2 \mathrm{X}$ were applied to the test bearing. Significant frequency dependent on the identified coefficients was observed.

Adams et al. [52] identified hybrid journal bearing stiffness, damping, and inertia coefficients using singlefrequency harmonic excitation. A two-rotor system was implemented to apply dynamic loads. The outer spindle was rotated independently of the inner rotor to provide different shaft orbits. Excitation frequencies of $4.2-20.8 \mathrm{~Hz}$ were produced. Forces and journal displacements were measured, and a least-squares solution of Eq. (5) was used to identify coefficients. Since 12 unknowns were identified, a minimum of three shaft orbits were required. No uncertainty analysis was provided for the identified coefficients. The amount of data in this report was limited for each of the rotordynamic coefficients, so an assessment of scatter could not be made. No comparisons to theoretical bearing coefficients were made. Additional results, including experimentally identified coefficients, were later provided [42].

Qiu and Tieu [83] identified the stiffness and damping coefficients for misaligned journal bearings. The excitation force applied to the bearing housing was provided with an eccentric-mass shaker. The frequency response functions for the rotor-bearing system including gyroscopic effects were measured, and data from two excitation frequencies were used with Eq. (7) to identify the bearing coefficients. A comprehensive uncertainty analysis, including the effects of measurement error, manufacturing error, force application, effects of vibration, and data quantization was performed. The theoretical coefficients fell inside the confidence interval for the experimentally identified coefficients.

Dmochowski [78] investigated the effect of flexible pivots on the frequency dependence of bearing dynamic coefficients. The shaft was held rigidly, and static and dynamic excitations were applied to the test bearing housing. Static loads were applied with tensioned cables, and dynamic loads were applied non-synchronously with electrodynamic shakers. Equation (1) was extended to include pad pivot equivalent stiffness and damping. Dynamic coefficient identification was accomplished with Eq. (5). Noise effects were reduced by using power spectral densities similar to methods developed by Dogan [39], Rouvas et al. [10], and Rouvas and Childs [11]. For the five-pad LBP bearing tested, the identified dynamic bearing coefficients were weakly dependent on frequency. The experimental results were used to validate a theoretical model. The theoretical model was applied to a five-pad LOP bearing. The predicted frequency dependence of the LBP bearing coefficients were attributed to the flexibility of the bearing pivots.

\subsection{Transient excitation}

The sinusoidal excitation methods discussed above apply forces at a single frequency, or in the case of a sine sweep, at discrete frequencies. Theoretically, a more efficient way to excite many frequencies is to apply a transient excitation, such as impulse or step loading, to the rotor bearing system. Bearing testers employing the transient excitation method have either used impulse hammers or released a static load to apply the impulse. In theory, all frequencies are applied simultaneously. However, with real impulse testers the selection of load release method, hammer mass, or hammer tip material results in the impact energy being preferentially applied to some frequencies or frequency range. Also, it is difficult to impart enough energy with impact forces to excite the higher frequency 
modes. Several replications are typically required to reduce the effects of noise on identified coefficients. The papers reporting results for transient excitation experiments are summarized in Table 4.

Morton [62] identified journal bearings by slowly applying a static load to a rotor. The applied load was suddenly released when a link supporting the load broke at a predetermined force level. The method was used on many turbomachines with journal diameters between $76.2-533.4 \mathrm{~mm}$. The method was applied to rotating machines in the field, so the rotors were flexible in general. The technique was developed for a rotor with the first natural frequency of $7.8 \mathrm{~Hz}$. Dynamic coefficients were identified using Eq. (6). The experimentally imposed time domain step function was converted to the frequency domain by a Fourier transform, and a frequency-domain correction factor was applied. A low-pass filter was also implemented to reduce the effects of noise. The corrected input force and rotor reactions were measured, and Eq. (5) was solved to identify the bearing coefficients. In this process, Eq. (5) was rearranged to isolate the flexible shaft degrees of freedom from the bearing degrees of freedom.

Nordmann and Schöllhorn [65] simultaneously identified the eight bearing stiffness and damping coefficients for two plain journal bearings by the use of the impulse method. The bearing dynamic coefficients for the two journal bearing were assumed to be identical. Impulse loading was applied to a rigid rotor using a hammer with accelerometer. The rotor was allowed to move within the bearings. The rotor response and input accelerations were measured and converted to the frequency domain. Equation (5) was solved to identify the bearing coefficients. The method was compared to the sinusoidal excitation method of Glienicke [40]. Differences between identified and theoretical coefficients were attributed to differences in the assumed temperature for oil viscosity. An isoviscous bearing model for theoretical coefficients was assumed.

Chang and Zheng [9] identified bearing stiffness and damping coefficients by the application of step forces. The tested elliptical journals were allowed to move over the rigidly held shaft. Static loads were applied to the bearing with use of an air bellows. Dynamic loading was applied by tightening a turnbuckle; then a step load was introduced by cutting a tension wire, releasing the bearing housing. The bearing displacement was measured using eddy current transducers, and acceleration was measured using accelerometers. Equation (5) was then used to perform the dynamic coefficient identification. Theoretical bearing coefficients were determined using a THD finite element model. The support bearings were not described in detail, so it is impossible to assess any effect these bearings may have had on results.

Kang and Jin [80] applied the model reference adaptive system (MRAS) to a plain journal bearing to identify the bearing dynamic stiffness and damping coefficients. The MRAS method considers the bearing-pedestal system on a per-bearing basis and places the equations of motion into state-space form. The reference model is required to be stable, controllable, and observable. The Lyapunov stability criteria are used in the iteration routine until results from the system model converge to the experimental data within an error tolerance. The technique was also applied to a rotor supported on two bearings. Excitation was applied with an impact hammer with force transducer. It was not clear from the paper whether the rotor or the bearings were excited. The bearing pedestals were identified using modal measurement techniques while the rotor was not rotating to provide baseline pedestal stiffness and damping. There were no theoretical values provided for oil-film coefficients.

Chan and White [73] identified the bearing coefficients for two tilting pad journal bearings simultaneously using the impact method. The test bearings supported a flexible angle shaft, which was part of a turbogenerator set. The dynamic load was applied to the rotor with an impact hammer, and input forces and output displacements were measured. A SDOF model of the rotor-bearing system in the form of Eq. (5) was used to identify bearing coefficients. Mass effects due to rotor flexibility were included as an effective mass matrix in the model. The effective mass is the modal mass associated with the first rotor critical speed. A set of experimental frequency response functions were fit to theoretical FRFs derived from Eq. (5) using a conventional least squares method. The phase data from the experimental FRFs was not used to perform the bearing parameter estimation. The cross-coupled coefficients were assumed to be negligible and were neglected. Ensemble averaging was used to smooth the data. One source of error was due to the two different methods for calculating the modal mass. The smaller modal mass value gave experimental results closer to the theoretical values for vertical coefficients. The larger modal mass gave experimental results closer to the theoretical values for horizontal coefficients. Other error sources included an assumed constant viscosity based on assumed oil mean temperature in the theoretical model, repeatability of impulse loading, and unknown oil flow conditions inside the bearing. The SDOF model was found to only be appropriate near the rotor critical speed, so the excitation frequency range was limited to values around the critical speed. 


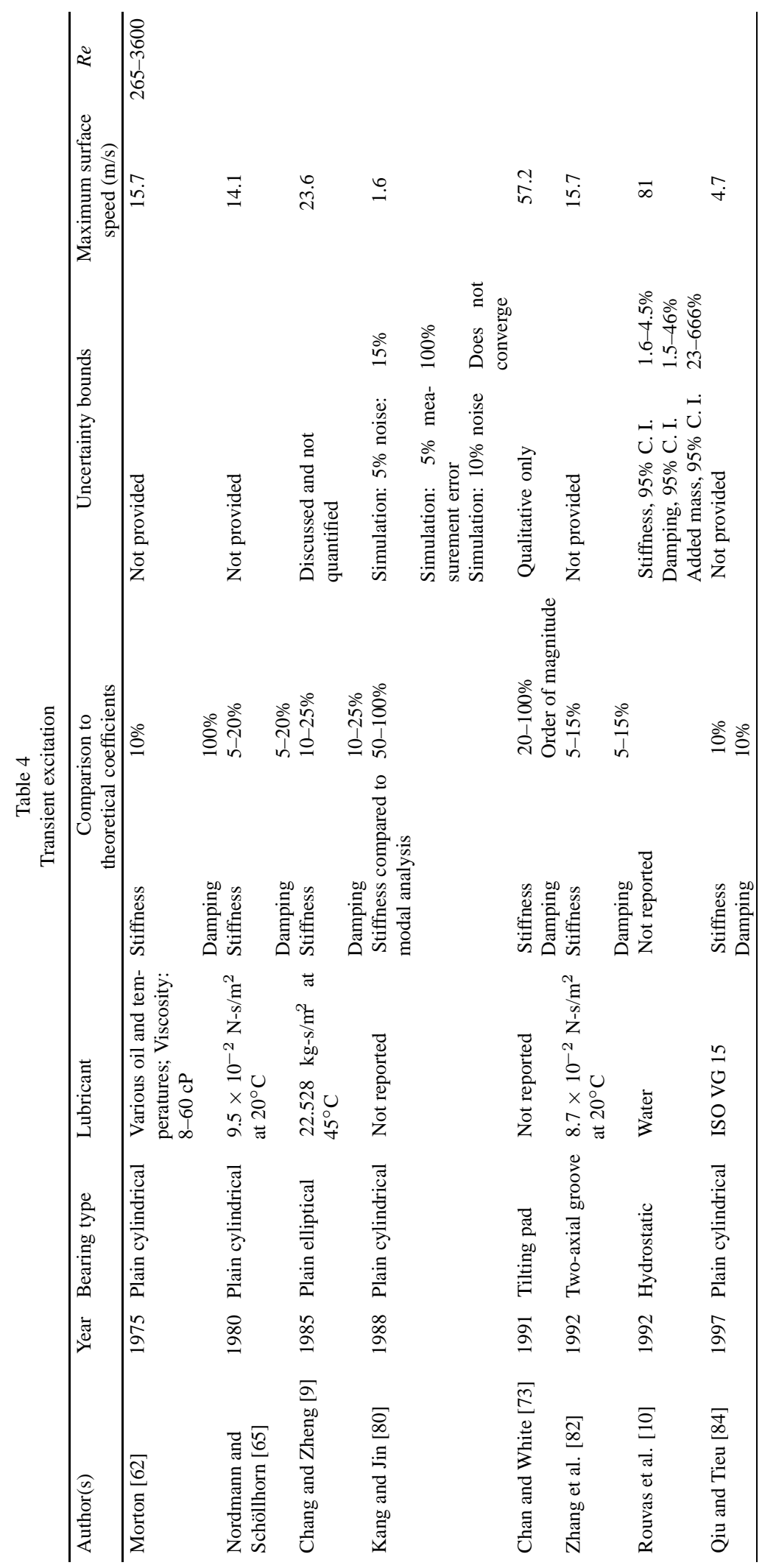


Zhang et al. [81,82] developed a method to identify bearing stiffness and damping coefficients when the rotor is flexible. A rotor supported in two bearings with a single disk was used for bearing identification. Frequency response functions were measured from impulse responses generated by a pendulum hammer impacting the disk. The rotor flexibility was treated as an equivalent stiffness, similarly to the shaft flexibility correction by Brockwell and Dmochowski [44]. The rotor-bearing equation of motion was presented as

$$
\left[\begin{array}{llll}
m_{d} & 0 & 0 & 0 \\
0 & m_{d} & 0 & 0 \\
0 & 0 & m_{s} & 0 \\
0 & 0 & 0 & m_{s}
\end{array}\right] \ddot{\mathbf{u}}+\left[\begin{array}{llll}
0 & 0 & 0 & 0 \\
0 & 0 & 0 & 0 \\
0 & 0 & c_{x x} & c_{x y} \\
0 & 0 & c_{y x} & c_{y y}
\end{array}\right] \dot{\mathbf{u}}+\left[\begin{array}{llll}
k_{r} & 0 & -k_{r} & 0 \\
0 & k_{r} & 0 & -k_{r} \\
-k_{r} & 0 & k_{r}+k_{x x} & k_{x y} \\
0 & -k_{r} & k_{y x} & k_{r}+k_{y y}
\end{array}\right] \mathbf{u}=\mathbf{f}
$$

Equation (5) was used to perform the identification. A weighted least-squares analysis was used to improve the numerical condition of the identification. Cross-correlation functions in the time domain were used to filter noise.

Qiu and Tieu [84] simultaneously identified the stiffness and damping coefficients for two bearings using impulse excitation. A rigid rotor held in the test bearings was tested. The bearing static load is due to the weight of the rotor, and dynamic loading is applied using an impact hammer. The bearing identification method included many replications, and Eq. (7) was solved using a least-squares analysis to estimate bearing coefficients. Only a single set of identified coefficients was presented. Differences between identified and theoretical coefficients were attributed to manufacturing differences and misalignment. The identified coefficients were used with a bell-shaped approximation to the impulse force to calculate theoretical FRFs. Agreement within 10 percent was obtained between measured and theoretical FRFs, indicating accurately identified bearing coefficients, but the level of correlation between measured and theoretical FRFs was not reported. The impulse also caused rotor motion of up to 20 percent of the bearing clearance, so non-linear bearing effects added to measurement error. Estimation accuracy was improved by measuring transfer functions at many frequencies near the shaft speed.

Rouvas et al. [10] presented a method for using power spectral densities to identify bearing coefficients. The measured forces, displacements, and accelerations were expressed in the frequency domain using the FFT. Then, by calculating the auto and cross spectral density functions, the effects of noise and turbulence on the identification were eliminated. The additional turbulence forces are assumed to be excitation-frequency independent. For bearing identification, Eq. (5) was rearranged as

$$
\left\{\begin{array}{l}
F_{x}-M A_{x} \\
F_{y}-M A_{y}
\end{array}\right\}=\left[\begin{array}{l}
H_{x x} H_{x y} \\
H_{y x} H_{y y}
\end{array}\right]\left\{\begin{array}{l}
U_{x} \\
U_{y}
\end{array}\right\}
$$

To reduce the effect of noise on the identification, Eq. (17) was expressed in terms of the autospectral and crossspectral densities and included forces from two independent excitations:

$$
\left[\begin{array}{ll}
G_{F_{x x} F_{x x}}-M G_{F_{x x} A_{x x}} G_{F_{y y} F_{y x}}-M G_{F_{y y} A_{y x}} \\
G_{F_{x x} F_{x y}}-M G_{F_{x x} A_{x y}} G_{F_{y y} F_{y y}}-M G_{F_{y y} A_{y y}}
\end{array}\right]=\left[\begin{array}{l}
H_{x x} H_{x y} \\
H_{y x} H_{y y}
\end{array}\right]\left[\begin{array}{l}
G_{F_{x x} U_{x x}} G_{F_{y y} U_{y x}} \\
G_{F_{x x} U_{x y}} G_{F_{y y} U_{y y}}
\end{array}\right]
$$

The rotor bearing system was excited with impact hammers. No comparison to theoretical values was made. Equation (5) was then used to identify stiffness, damping, and inertia coefficients. The uncertainty was presented as standard deviations in the identified coefficients.

\subsection{Pseudorandom excitation}

A pseudorandom excitation is applied by simultaneously applying forces to the rotor-bearing system at many frequencies. The pseudorandom signal is composed of sine waves at different frequencies and different phases. The amplitudes and phases are adjusted so that the peak amplitude of the force signal does not cause large vibrations that violate the linearity assumption for bearing coefficients. The advantage to this simultaneous frequency excitation is that the bearing response also has simultaneous frequency content, and frequency response functions can be experimentally identified from short time samples of the data. This approach is less time-consuming than a sine sweep, where each frequency is applied separately. The system must come to equilibrium at each discrete frequency of the sine sweep, increasing the total time required. The short time required to obtain data with pseudorandom excitation also reduces the possibility that changes in lubricant viscosity due to temperature, dimensional changes, 
and other effects will affect identified coefficients. Psuedorandom excitation also has advantages over impulse testing. The spectral representation of the pseudorandom signal only contains information at discrete frequencies, so the representation of is simpler than the spectral representation of a step function. The papers describing results for pseudorandom excitation are summarized in Table 5.

Dogan et al. [39] identified bearing dynamic coefficients for a plain cylindrical journal bearing using a pseudorandom binary sequence excitation profile. Excitation frequencies ranged from 6-120 Hz. Dynamic loads were applied using piezoelectric load washers attached to rods. The rods were attached to the bearing housing. The static loading method was not reported. Frequency response functions were measured experimentally from spectral density functions similar to Eq. (18). The coefficients were identified using a non-linear steepest descent curve fit. Non-dimensional results were reported

Burrows and Sahinkaya [14] developed a method employing a multi-frequency forcing signal. Although applicable to fluid film bearings, experimental results for a squeeze film damper were reported in this paper. The methods for static and dynamic loading of the damper were not reported. Equation (7) was used to identify squeeze-film damper coefficients in the frequency domain. The effects of colored and white noise on measured forces and displacements on the identified coefficients were investigated with a simulation example. Stiffness coefficients were neglected and only damping coefficients were identified. The damping coefficients were identified within 5-10 percent of theory except for $C_{y x}$, compared to a time-domain method that resulted in up to 100 percent difference between experiment and theory. The errors in $C_{y x}$ were attributed to low x-axis motions compared to y-axis motion.

Rouvas and Childs [11] took the power spectral density method developed by Rouvas et al. [10] and applied pseudorandom excitations instead of impact forces. The force content was based on the superposition of 41 sinusoids to produce a pseudo-random excitation. The phase of the individual force components were optimized to keep the overall maximum force amplitudes low.

The same approach was implemented in a test apparatus described by Childs and Hale [15]. The test rig consisted of a rotor held in rolling element bearings, with a test hydrostatic bearing allowed to move over the shaft. Heated water was used as the lubricant. Orthogonal shakers were used to excite the bearing housing with pseudorandom input. Spectral density averaging was used to smooth the experimental frequency response functions. The bearing parameters were then identified from Eq. (18). Uncertainty analysis on the identified coefficients was performed based upon the method given in [5].

The rig and identification approach has been used to identify bearing coefficients for roughened-land hybrid bearings [58], orifice-compensated hybrid bearings [53,54,59], and angled injection and near-tangential-injection hybrid bearings [26,27,48,84]. Laurant and Childs [26] reported the mass coefficients in terms of an effective stiffness and damping, defined as

$$
\begin{aligned}
& k_{e f f}=k_{x x}+c_{x y} \Omega-m_{x x} \Omega^{2} \\
& c_{e f f}=c_{x x}-\frac{k_{x y}}{\Omega}+m_{x y} \Omega
\end{aligned}
$$

Rodriguez and Childs [49] and Al-Ghasem and Childs [2] reported excitation-frequency-dependent results for fivepad flexible-pivot bearings. The rotor was held in rolling element bearings, and excitation forces were applied to the bearing housing. The frequency response functions were calculated using methods adapted from [15]. The key difference with tilting pad bearings is that the fluid structure interaction forces are now excitation frequency dependent, so the assumptions that went into using uncorrelated power spectral densities were no longer valid. As a result, Eq. (5) was used to identify the bearing coefficients. The theoretical basis for including mass coefficients in the bearing identification was based on Reinhardt and Lund [24]. For a short rotor operated with bearing Reynolds number between 500-750, the lubricant inertia was found to be of the same order of the rotor inertia. Bearing mass coefficients are then stated in the paper to be significant for evaluating rotordynamic performance. The theoretical bearing dynamic coefficients were calculating using an isoviscous bulk flow model.

\subsection{Known/Additional unbalance excitation}

Some researchers have focused on excitation methods that could be applied in the field with minimal additional equipment. In addition to the impulse/step functions previously discussed, using a known or additional unbalance 


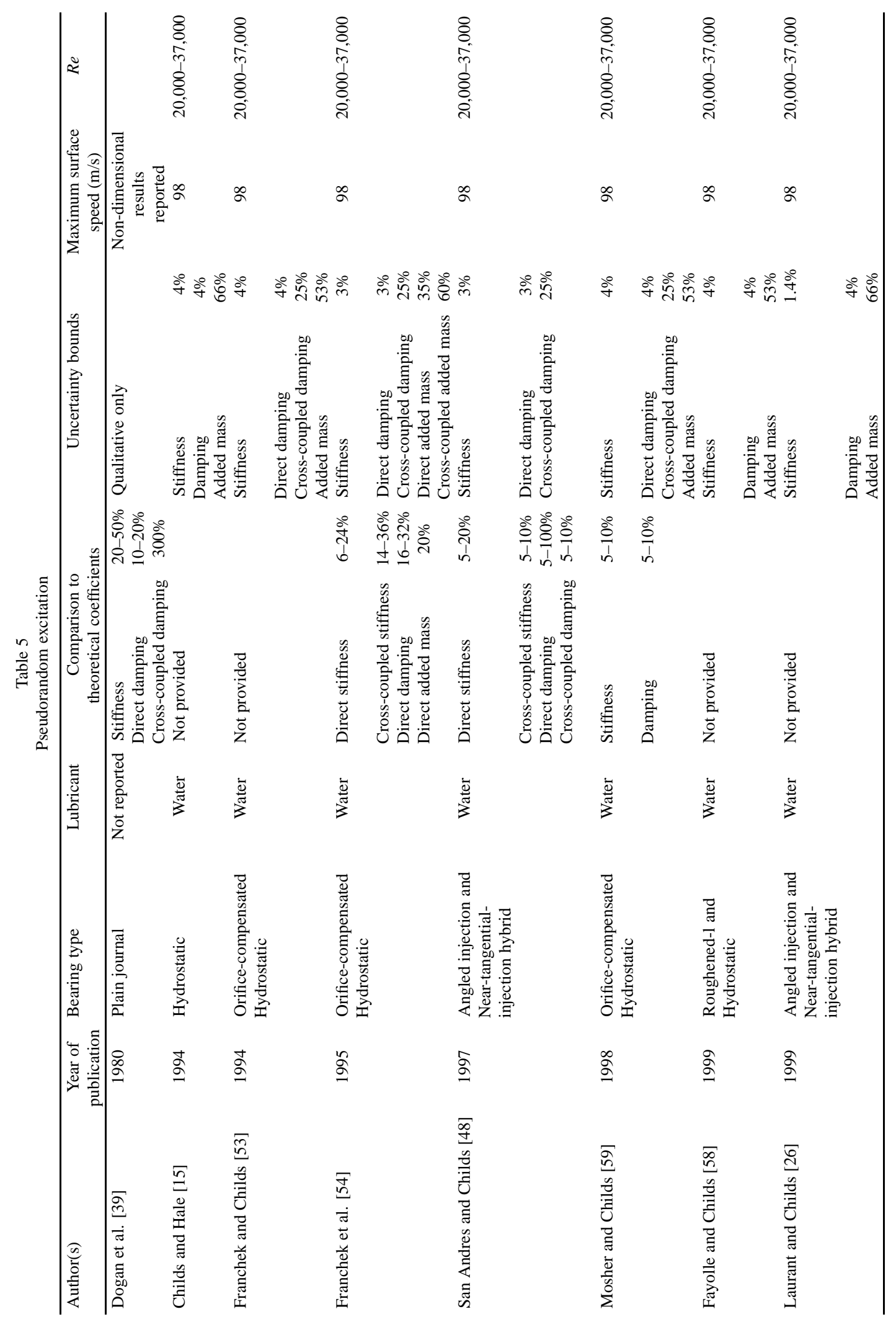




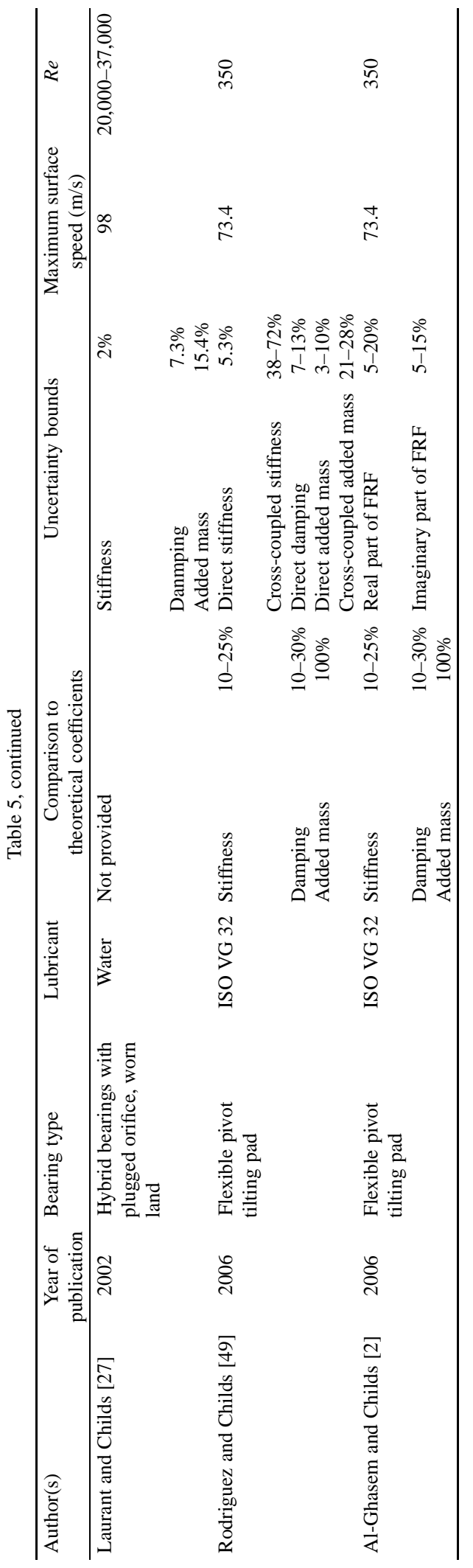


on the rotor has been used in identification. Although easily implemented, the method is limited to synchronous excitation of the test rotor unless a concentric rotor arrangement is used. The use of unbalance typically leads to circular whirl orbits of the shaft, which usually results in poor numerical conditioning of the matrix inversions and least squares formulations of Eqs (4)-(6). The papers reporting results for unbalance excitation are summarized in Table 6.

Yanabe et al. [72] identified bearing stiffness coefficients using incremental static loading and bearing damping coefficients using force and displacement measurements resulting from the rotor residual unbalance. The tested bearing was a plain journal bearing. An unbalance disc was included for damping measurements, but was removed for stiffness measurements to reduce the effect on unbalance on results. Equations (7)-(8) were used to identify the bearing stiffness due to the incremental loading. The stiffness results from the influence coefficient measurement were used with Eq. (7) to identify damping coefficients. The measured experimental results were compared to theoretical values calculated with an isoviscous bearing model, using a finite difference approach.

Tieu and Qiu [1] proposed a method using multiple unbalance weights for identification of the stiffness and damping coefficients for two plain journal bearings. The rotor was held in the journal bearings to be identified. The method was based on a rigid rotor model, with responses from multiple unbalance runs measured in the frequency domain. Equation (7) was used to identify bearing coefficients. One of the simplifications to the method was to use the average bearing clearance between the two journals in calculations. The identified bearing coefficients were compared to theoretical coefficients that were calculated using an isoviscous lubricant bearing model. Tieu and Qiu reported a small experimentally measured temperature increase of $1^{\circ} \mathrm{C}$ from oil inlet to oil outlet for these bearings, so an isoviscous assumption was appropriate in this case. Even with between-bearing clearance averaging, the identified coefficients were within 5-10 percent of theoretical values calculated by Lund and Thomsen [43].

Pettinato and Choudhury [6] identified bearing coefficients for tilting-pad bearings with unbalance excitation. Incremental loading was used to identify bearing stiffness. Additional unbalance was added at the test end bearing for damping estimation. At a given running speed, the level of unbalance was changed to produce different dynamic loads on the bearings. Equation (8) was used to measure the stiffness coefficients. The exact method for estimating damping coefficients was not reported. The effect of pivot stiffness on overall bearing coefficients was also measured. Uncertainty and scatter increased with increasing running speed. Experimentally identified coefficients were compared to an isoviscous bearing model. The difference between identified and theoretical stiffness coefficients became larger as bearing static load was increased. Pads and supports were considered rigid, and the bearing pads were considered to be massless.

Tiwari et al. [67] identified the speed-dependent bearing parameters for a flexible rotor-bearing system in the presence of measurement noise using unbalance excitation. The experimental rotor was flexible in the operating region and was modeled using finite elements. The experimental rotor was held in rolling element bearings supported by springs and dampers, to simulate a fluid-film bearing. The free-free rotor model was confirmed experimentally. The bearing mass, stiffness, and damping coefficients were identified in the frequency domain using Eq. (7), with additional degrees of freedom to represent the rotor flexibility. Row and column operations were performed on Eq. (7) to isolate the bearing degrees of freedom. When the bearings were isotropic or nearly isotropic, a regularized least-squares formulation [3] was applied to improve the numerical condition of the problem. The conventional least squares formulation applied to Eq. (7) is

$$
\min \|\mathbf{U} \beta-\mathbf{f}\|_{2}
$$

When this was applied to a rotor with circular or nearly circular orbits, the identification problem became ill-posed. The condition of the least squares was improved by a regularization matrix:

$$
\min \left\{\|\mathbf{U} \beta-\mathbf{f}\|_{2}^{2}+\lambda^{2}\|\mathbf{L} \beta\|_{2}^{2}\right\}
$$

The parameter $\lambda$ was optimized to minimize the individual norms $\|\mathbf{H u}-\mathbf{f}\|_{2}$ and $\|\mathbf{L u}\|_{2}$.

The regularizing matrix $\mathbf{L}$ is usually the identity matrix, but was selected in this paper to minimize the squared difference between horizontal and vertical parameters for nearly isotropic bearings. Numerical studies performed by the authors showed that the choice of regularization matrix resulted in a biased estimate of bearing stiffness. The direct stiffness estimate was biased towards the higher stiffness. The amount of bias error introduced by the regularization was not quantified. 


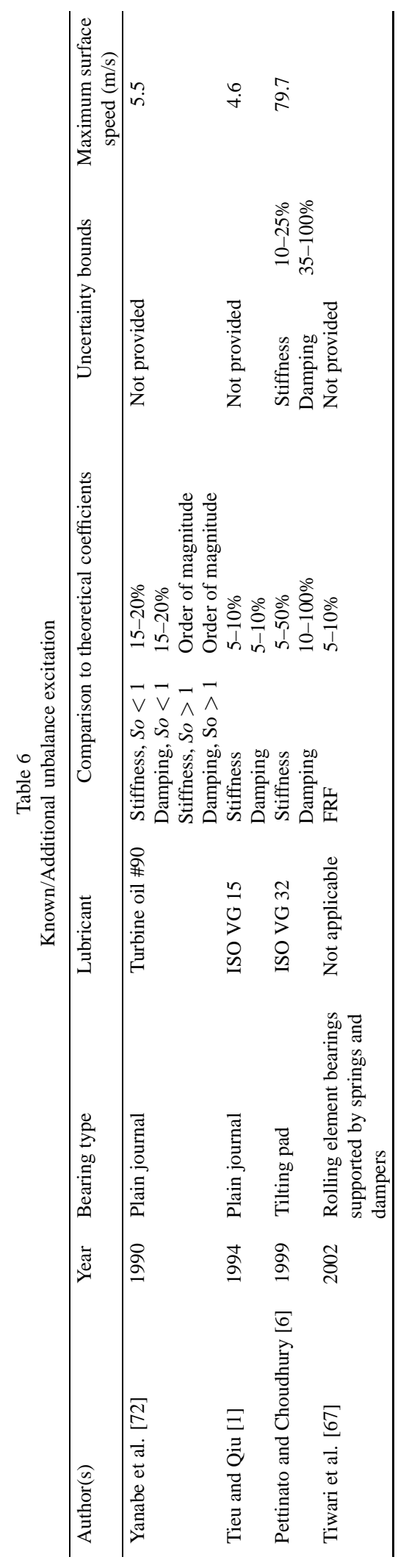




\section{Non-contact excitation}

A recent development in the identification of bearing dynamic coefficients is the use of non-contact force actuators to excite the rotor bearing system. The exciters, typically active magnetic bearings or non-contact electromagnetic shakers, reduce the amount of uncertainty in measurement by reducing the additional dynamics inherent with incontact excitation methods. Non-contact exciters allow flexibility in force application, so either the sinusoidal or pseudorandom excitation techniques can be used. One of the challenges with the non-contact exciters is estimating the input force to the rotor bearing system. The papers reporting results for non-contact excitation methods are summarized in Table 7.

Arumugam et al. [55] identified coefficients for cylindrical and tilting pad bearings using a non-contact electromagnetic exciter. The experimental flexible was supported by two bearings, with a single disc between the bearings. One support bearing was the test bearing, and the other support bearing was a rolling-element bearing. Dynamic forces were applied to the rotor between $0-100 \mathrm{~Hz}$ using a unidirectional sine sweep perturbation. The displacement was measured near the test bearing to measure the associated FRF. Only two FRFs were measured for the tilting pad bearings, and four FRFs were measured for the plain journal bearing. The free-free FRFs for the rotor were found using the finite element method, without experimental verification. The frequency response function of the rotor bearing system was then found experimentally, and bearing coefficients were calculated with a least squares curve solution to Eq. (7). The experimentally identified stiffness and damping showed some scatter in the data. The highest amount of scatter occurred for the load-between-pad tilting pad cases and the least amount of scatter for the plain journal bearing. While not stated explicitly in the paper, it is assumed in this work that the synchronously reduced bearing coefficients were identified for the tilting pad bearings. Scatter was attributed to changes in oil viscosity, and excitation of forward and backward modes. Another tilting pad bearing source of error not included in this paper were the additional coefficients due to the degrees of freedom introduced by pad motion. No comparison was made to theoretical coefficients, but the experimentally identified coefficients were used to calculate the system FRF, which was compared to the experimentally identified system FRF.

The rig was also used to identify bearing coefficients for misaligned plain and three lobe journals [56,57]. With the misaligned bearings, there were 24 additional stiffness and damping coefficients associated with moments in the bearing, which could not all be identified using the FRF method. A simulation study indicated that the error introduced on the force coefficients by not identifying the additional moment bearing coefficients was a maximum of 0.7 percent. However, the rotor was held in two cylindrical journal bearings at either end, with the test bearing in the center of the rotor for this test set up. Two discs were included on the rotor. The non-contact electromagnetic exciter applied dynamic loads to one of the discs. Curve fitting of the experimental FRFs prior to identification was used to reduce noise effects. The identified trends reported in the paper were that vertical stiffness and cross-coupled stiffness $k_{y x}$ increased with misalignment, while horizontal stiffness and cross-coupled stiffness $k_{x y}$ decreased. The identified principal damping increased with misalignment, while no trend was discernable with cross-coupled damping. An uncertainty analysis similar to Kostrzewsky and Flack [29] was performed.

Tilting pad journal bearings with small L/D ratios were also identified with this approach [16]. The experimentally identified bearing coefficients were compared to theoretically calculated values calculated from a TEHD bearing model. There was some scatter in the data, especially for the $\mathrm{L} / \mathrm{D}=0.4$ case, but the identified coefficients showed good agreement with the TEHD model.

Knopf and Nordmann [23] used magnetic bearings as actuators to identify the bearing stiffness, damping, and inertia coefficients for turbulent plain journal bearings. The rotor was held with the magnetic bearings, with the test bearing in the center. The arrangement is shown in Fig. 5. Input forces to the shaft were calculated from magnetic fluxes measured with Hall sensors. A stepped sine forcing function was applied to the bearing non-synchronously. Bearing identification was carried out in the time domain by a least-squares fit of an artificial harmonic signal to Eq. (5). Linearity of the experiment was imposed by limiting displacement amplitudes if higher harmonic levels reached a tolerance. Measured forces were consistent with a turbulence model proposed by Constantinescu [77]. The experimental coefficients were presented, and the experimental So was compared to the traditional laminar So and the So with turbulence correction. The stability of the journal bearing was also assessed using a turbulent bearing model. The total uncertainty in identified bearing coefficients and experimental So were not reported. The 


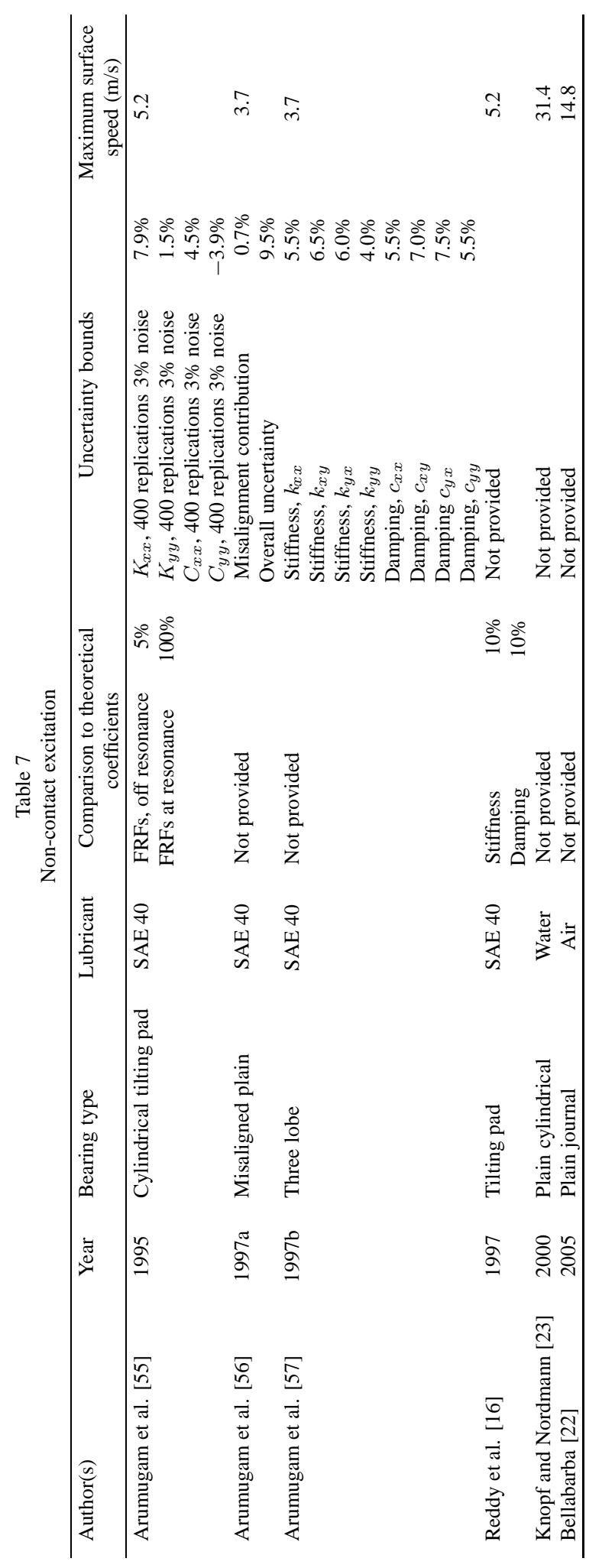




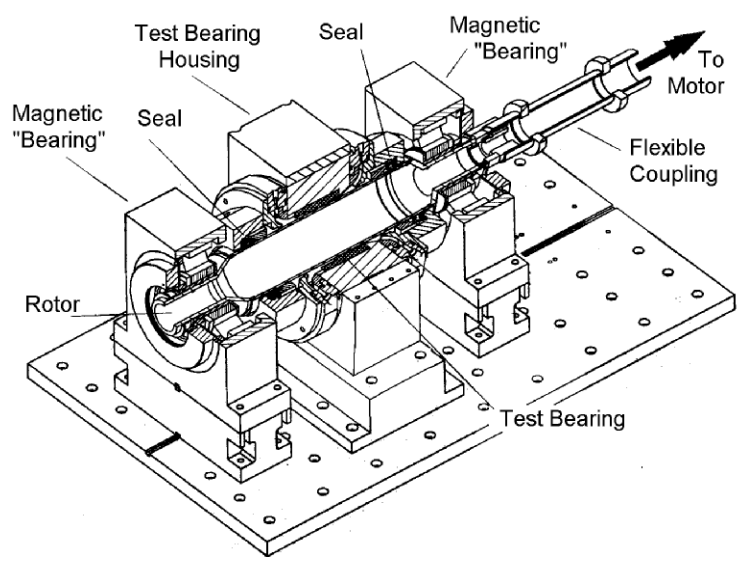

Fig. 5. Knopf and Nordmann [23] test rig.

identification resulted in negative direct mass coefficients for eccentricities between $0.55-0.75$. Negative direct masses are not predicted by any theoretical bearing model and indicate a need to review the identification method.

Bellabarba et al. [22] identified stiffness and damping coefficients for air bearings using active magnetic bearings as force exciters. The air bearing tested was supported by load cells, and the reaction forces of the bearing shell were measured for identification. Rotor tilt was not considered. Displacement and force measurements were converted to the frequency domain, and FRF curve fits of Eq. (5) using an Instrumental Variable Filter were used to identify bearing stiffness and damping coefficients. Care was taken to properly align the rotor within the magnetic bearings and the air bearing. The additional dynamics introduced by the load cells was not directly addressed.

\section{Conclusions}

The general trends in experiments have reflected the requirements of the turbomachinery industry. As journal surface speeds have increased, the experiments have reflected those changes. As journal speeds result in fully turbulent lubricants, the need for accurate bearing coefficients increases.

Bearing identification generally results in noisy data. The industrial trend towards fully turbulent bearing lubricants increases the amount of noise in the identification problem. Trends in improving quality of data include the development of filtering and conditioning techniques such as the use of spectral density averaging, improving the numerical condition of matrix inversion through careful experimentation, and least-squares regularization to improve the quality of the data. More recent experimenters have also quantified the confidence bounds on the identified coefficients.

Many researchers have used isoviscous bearing models to calculate theoretical bearing coefficients. However, the experiments often showed significant temperature gradients in the bearing. Viscosity is a strong function of temperature in oil-based lubricants, so the isoviscous analysis is violated. More recent bearing experiments have recognized the necessity of THD and TEHD models. However, agreement between theory and experiment for bearing coefficients is seldom better than 10-20 percent.

One of the general discrepancies between predicted and measured coefficients is that the predicted damping coefficients are overpredicted. Some of the discrepancy could be resolved by measurement of parameters such as hot running bearing clearances, so that accurate values can be used for model reconciliation. Some experimenters have attempted to quantify these effects on coefficients, including flexibility of tilting pad pivots and the dynamics introduced by flexibility of the bearing shell and supports.

The non-contact excitation methods hold promise in improving the overall quality of measured data. The advantage of removing the additional dynamics from the traditional contact excitation methods can help reduce measurement uncertainty, which will result in smaller confidence bounds on identified coefficients. 


\section{Acknowledgement}

The interest and the financial support of the industrial members of the Rotating Machinery and Controls (ROMAC) Laboratory of Department of Mechanical and Aerospace Engineering at University of Virginia are gratefully acknowledged.

\section{Nomenclature}

$A-$ Bearing housing acceleration

$D$ - journal diameter

$G_{i j}$ - single-sided cross/autospectral density function

$\mathbf{H}$ - complex multiple-input multiple-output (MIMO) frequency response function (impedance)

$F_{x}, F_{y}$ - external input force to bearing/rotor

$J_{p n}-$ mass moment of inertia of bearing pads

$M-$ mass of excited bearing housing/rotor

$N$ - rotor running speed, rpm

$L-$ journal length

$R$ - journal radius

Re - Reynolds number, $c_{p} \Omega \mathrm{D} / \nu$

So - Sommerfeld number, $\mu N D L /(2 \pi W) \cdot\left(R / c_{p}\right)^{2}$

$\mathbf{U}$ - matrix of measured rotor lateral displacements/accelerations

$W$ - bearing load

$\mathbf{Z}$ - complex MIMO frequency response function (mobility)

$a_{i j}$ - bearing influence coefficient

$\mathbf{a}$ - bearing influence coefficient matrix

$c_{e q}$ - equivalent bearing direct damping

$c_{i j}$ - bearing damping coefficient

$c_{p}$ - bearing pad clearance

f - vector of input forces

$i, j-$ load/displacement/velocity/acceleration direction index

$k_{e q}$ - equivalent bearing direct stiffness

$k_{i j}$ - bearing stiffness coefficient

$k_{s}-$ shaft stiffness

$m_{d}$ - disk mass

$m_{i j}$ - bearing added mass coefficient

$m_{s}-$ shaft mass

$\mathbf{u}, \dot{\mathbf{u}}, \ddot{\mathbf{u}}$ - displacement, velocity, acceleration vector

$x, \dot{x}, \ddot{x}$ - bearing/rotor horizontal displacement, velocity, acceleration

$y, \dot{y}, \ddot{y}$ - bearing/rotor vertical displacement, velocity, acceleration

$\Omega$ - frequency of exciting force

$\delta_{n}, \dot{\delta}_{n}, \ddot{\delta}_{n}$ - bearing pad angular displacement, velocity, acceleration about pivot

$\varepsilon-$ bearing eccentricity ratio

$\lambda$ - regularized least squares parameter

$\nu$ - lubricant kinematic viscosity

$\phi_{0}$ - bearing attitude angle

$\omega$ - rotational speed of rotor 


\section{References}

[1] A.K. Tieu and Z.L. Qiu, Identification of sixteen dynamic coefficients of two journal bearings from experimental unbalance responses, Wear 177 (1994), 63-69.

[2] A.M. Al Ghasem and D.W. Childs, Rotordynamic Coefficients Measurements Versus Predictions for a High-Speed Flexure-Pivot Tilting Pad Bearing (Load-Between-Pad Configuration), J Engr Gas Turbines Pwr 128 (2006), 896-906.

[3] A.N. Tikhonov and V.Y. Arsenin, Solutions of Ill-Posed Problems, V.H. Winston and Sons, Washington, DC, 1977.

[4] API 617, Axial and Centrifugal Compressors and Expander-compressors for Petroleum, Chemical and Gas Industry Services, Seventh Edition, 2002

[5] ASME PTC 19.1, Test Uncertainty, Instruments, and Apparatus, 1998.

[6] B. Pettinato and P. de Choudhury, Test Results for Key and Spherical Pivot Five-Shoe Tilt pad Journal Bearings - Part II: Dynamic Measurements, Tribol Trans 42 (1999), 675-680.

[7] B. Pettinato, R.D. Flack and L.E. Barrett, Test results for a highly preloaded three-lobe journal bearing - Effect of load orientation on static and dynamic characteristics, Lubric Engin 57 (2001), 23-30.

[8] B.C. Pettinato, R.D. Flack and L.E. Barrett, Effects of excitation frequency and orbit magnitude on the dynamic characteristics of a highly preloaded three-lobe journal bearing, Tribol Trans 44 (2001), 575-582.

[9] C. Chang and P. Zheng, Experimental determination of the stiffness and damping coefficients of fluid film bearings by means of step forces, Tribol Internat 18 (1985), 81-91.

[10] C. Rouvas, B.T. Murphy and R.K. Hale, Bearing parameter identification using power spectral density methods, in: Proc. 5th International Conference on Vibrations in Rotating Machinery, IMechE, Bath, UK, 1992, 297-303.

[11] C. Rouvas and D.W. Childs, A Parameter Identification Method for the Rotordynamic Coefficients of a High Reynolds Number Hydrostatic Bearing, J Vibr Acoust 115 (1993), 264-270.

[12] C.H. Cloud, Stability of Rotors Supported by Tilting Pad Journal Bearings, Ph.D. Thesis, University of Virginia, 2007.

[13] C.M. Myllerup, J. Tonnesen and J.W. Lund, On the discrepancies between experiment and theory for a cylindrical fluid-film journal bearing considering steady-state and dynamic characteristics, in: Proc. 5th International Conference on Vibrations in Rotating Machinery, IMechE, Bath, UK, 1992, 1-6.

[14] C.R. Burrows and M.N. Sahinkaya, Frequency-Domain Estimation of Linearized Oil-Film Coefficients, J Lubric Tech 104 (1982), $210-215$.

[15] D. Childs and K. Hale, A Test Apparatus and Facility to Identify the Rotordynamic Coefficients of High-Speed Hydrostatic Bearings, $J$ Tribol 116 (1994), 337-344.

[16] D.S.K. Reddy, S. Swarnamani and B.S. Prabhu, Experimental investigation on the performance characteristics of tilting pad journal bearings for small L/D ratios, Wear 292 (1997), 33-40.

[17] D.V. Taylor, G.J. Kostrzewsky, L.E. Barrett and R.D. Flack, Measured performance of a highly preloaded three-lobe journal bearing - part II: dynamic characteristics, Tribol Trans 38 (1995), 707-713.

[18] D.W. Parkins, Theoretical and Experimental Determination of the Dynamic Characteristics of a Hydrodynamic Journal Bearing, $J$ Lubr Technol 101 (1979), 129-139.

[19] D.W. Parkins, Measured Characteristics of a Journal Bearing Oil Film, J Lubr Technol 103 (1981), $120-125$.

[20] D.W. Parkins, Measurement of Oil Film Journal Bearing Damping Coefficients - An Extension of the Selected Orbit Technique, J Tribol 117 (1995), 696-701.

[21] D.W. Parkins and D. Horner, Tilting Pad Journal Bearings - Measured and Predicted Stiffness Coefficients, Tribol Trans 36 (1993), 359-366.

[22] E. Bellabarba, S. Diaz and V. Rastelli, A Test Rig for Air Bearings Rotordynamic Coefficients Measurement, in: Proc. ASME Turbo Expo 2005, Reno-Tahoe, NV, 2005, 833-839.

[23] E. Knopf and R. Nordmann, Identification of the dynamic characteristics of turbulent journal bearings using active magnetic bearings, in: Proc. $7^{\text {th }}$ International Conference on Vibrations in Rotating Machinery, IMechE, Nottingham, UK, 2000, 381-390.

[24] E. Reinhardt and J.W. Lund, The Influence of Fluid Inertia on the Dynamic Properties of Journal Bearings, J Lubric Tech 97 (1975), $159-167$.

[25] E.E. Swanson and R.G. Kirk, 1997, Survey of Experimental Data for Fixed Geometry Hydrodynamic Journal Bearings, J Tribol 119 (1989), 704-710

[26] F. Laurant and D.W. Childs, Rotordynamic Evaluation of a Near-Tangential-Injection Hybrid Bearing, J Tribol 121 (1999), 886-891.

[27] F. Laurant and D.W. Childs, Measurements of Rotordynamic Coefficients of Hybrid Bearings With (a) a Plugged Orifice, and (b) a Worn Land Surface, J Engr Gas Turbines Pwr 124 (2002), 363-368.

[28] G. Diana, D. Borgese and A. Dufour, Experimental and Analytical Research on a Full Scale Turbine Journal Bearing, in: Proc. 22d International Conference on Vibrations in Rotating Machinery, IMechE, Cambridge, UK, 1980, 355-360.

[29] G.J. Kostrzewsky and R.D. Flack, Accuracy evaluation of experimentally derived dynamic coefficients, of fluid film bearings. Part I. Development of Method, Tribol Trans 33 (1990), 105-114.

[30] G.J. Kostrzwesky, R.D. Flack and L.E. Barrett, Theoretical and experimental dynamic characteristics of a highly preloaded three-lobe journal bearing, Tribol Trans 41 (1998), 392-398.

[31] G.J. Kostrzewsky, R.D. Flack and L.E. Barrett, Comparison between measured and predicted performance of a two-axial-groove journal bearing, Tribol Trans 39 (1996), 571-578.

[32] G.J. Kostrzewsky, D.V. Taylor and R.D. Flack, Experimental determination of the dynamic characteristics of a two-axial groove journal bearing, Tribol Trans 37 (1994), 534-542.

[33] H. Kanki, Y. Ozawa, O. Takanobu and T. Kawakami, Study on the Dynamic Characteristics of a Water-Lubricated Pump Bearing, Bulletin of JSME 29 (1986), 1824-1829. 
[34] H. Sakakida, S. Asatsu and S. Tasaki, The static and dynamic characteristics of 23 inch (584.2 mm) diameter journal bearing, in: Proc. $5^{\text {th }}$ International Conference on Vibration in Rotating Machinery, IMechE, Bath, UK, 1992, 351-357.

[35] H.C. Ha and S.H. Yang, Excitation Frequency Effects on the Stiffness and Damping Coefficients of a Five-Pad Tilting Pad Journal Bearing, J Tribol 121 (1999), 517-522.

[36] I. Matsumoto, J. Esaki, T. Furukawa and S. Taniguchi, Oil Film and Vibration Characteristics of Offset-Halves Journal Bearing, in: Proc. $3^{\text {rd }}$ International Conference on Rotordynamics, M. Lalanne and R. Henry, eds, IFToMM, Lyon, France, 1990 , pp. 105-110.

[37] I. Santos, On the Adjusting of the Dynamic Coefficients of Tilting-Pad Journal Bearings, Tribol Trans 38 (1995), $700-706$.

[38] I.F. Santos, Theoretical and Experimental Identification of the Stiffness and Damping Coefficients of Active Tilting-Pad Journal Bearings, in: Proc. Identification in Engineered Systems, M.I. Friswell and J.E. Mottershead, eds, University of Wales, Swansea, UK, 1996, pp. 783-792.

[39] I.U. Dogan, J.S. Burdess and J.R. Hewit, Identification of Journal Bearing Coefficients using a Pseudo-Random Binary Sequence, in: Proc. Vibrations in Rotating Machinery: $2^{\text {nd }}$ International Conference, IMechE, Cambridge, UK, 1980, $277-281$.

[40] J. Glienicke, Experimental Investigation of the Stiffness and Damping Coefficients of Turbine Bearings and their Application to Instability Prediction, Proc IMechE 181 (1966-1967), 116-129.

[41] J.R. Mitchell, R. Holmes and H. Van Ballegooyen, Experimental Determination of a Bearing Oil-Film Stiffness, Proc. IMechE 180 (1965-1966), 90-96.

[42] J.T. Sawicki, R.J. Capaldi and M.L. Adams, Experimental and Theoretical rotordynamic Characteristics of a Hybrid Journal Bearing, $J$ Tribol 119 (1997), 132-142.

[43] J.W. Lund and K.K. Thomsen, A calculation method and data for the dynamic coefficients of oil-lubricated journal bearings, in: Topics in fluid film bearing and rotor bearing system design and optimization: Proc. ASME Design Engineering Conference, S.M. Rohde, P.E. Allaire and C.J. Maday, eds, ASME, Chicago, IL, 1978, pp. 1-28.

[44] K. Brockwell and W. Dmochowski, Experimental determination of the journal bearing oil film coefficients by the method of selective vibration orbits, in: ASME Conference on Mechanical Vibration and Noise (12 $\left.2^{\mathrm{h}}\right)$, T.S. Sankar et al., eds, Montréal, Québec, 1989, pp. 251-259.

[45] K. Brockwell, D. Kleinbub and W. Dmochowski, Measurement and Calculation of the Dynamic Operating Characteristics of the Five Shoe, Tilting Pad Journal Bearing, Tribol Trans 33 (1990), 481-492.

[46] K.D. Wygant, L.E. Barrett and R.D. Flack, Influence of pad pivot friction on tilting-pad journal bearing measurements - Part II: dynamic coefficients, Tribol Trans 42 (1999), 250-256.

[47] K.D. Wygant, R.D. Flack and L.E. Barrett, Measured performance of tilting-pad journal bearings over a range of preloads - Part II: Dynamic operating conditions, Tribol Trans 47 (2004), 585-593.

[48] L. San Andres and D. Childs, Angled Injection - Hydrostatic Bearings Analysis and Comparison to Test Results, J Tribol 119 (1997), 179-187.

[49] L.E. Rodriguez and D.W. Childs, Frequency Dependency of Measured and Predicted Rotordynamic Coefficients for a Load-on-Pad Flexible-Pivot Tilting-Pad Bearing, J Tribol 128 (2006), 388-395.

[50] M. He, Thermoelastohydrodynamic Analysis of Fluid Film Journal Bearings, Ph.D. thesis, University of Virginia, 2003.

[51] M.J. Goodwin, Experimental Techniques for Bearing Impedance Measurements, J Engin Indus 113 (1991), $335-342$.

[52] M.L. Adams, J.T. Sawicki and R.J. Capaldi, Experimental determination of hydrostatic journal bearing rotordynamic coefficients, in: Proc. 5th International Conference on Vibrations in Rotating Machinery, IMechE, Bath, UK, 1992, 365-374.

[53] N.M. Franchek and D.W. Childs, Experimental Test Results for Four High-Speed, High-Pressure, Orifice-Compensated Hybrid Bearings, J Tribol 116 (1994), 147-153.

[54] N.M. Franchek, D.W. Childs and L. San Andres, Theoretical and Experimental Comparisons for Rotordynamic Coefficients of a HighSpeed, High-Pressure, Orifice-Compensated Hybrid Bearing, J Tribol 117 (1995), 285-290.

[55] P. Arumugam, S. Swarnamani and B.S. Prabhu, Experimental Identification of Linearized Oil Film Coefficients of Cylindrical and Tilting Pad Bearings, J Eng For Gas Turbines and Power 117 (1995), 593-599.

[56] P. Arumugam, S. Swarnamani and B.S. Prabhu, An Experimental Investigation on the Static and Dynamic Characteristics of Journal Bearings Under the Influence of Twisting Misalignment, J Tribol 119 (1997), 188-192.

[57] P. Arumugam, S. Swarnamani and B.S. Prabhu, Effects of journal misalignment on the performance characteristics of three-lobe bearings, Wear 206 (1997), 122-129.

[58] P. Fayolle and D.W. Childs, Rotordynamic Evaluation of a Roughened-Land Hybrid Bearing, J Tribol 121 (1999), $133-138$.

[59] P. Mosher and D.W. Childs, Theory Versus Experiment for the Effects of Pressure Ratio on the Performance of an Orifice-Compensated Hybrid Bearing, J Vibr Acoust 120 (1998), 930-936.

[60] P.E. Allaire and R.D. Flack, Journal Bearing Design for High-Speed Turbomachinery, in: Proc. International Conference on Bearing Design, Historical Aspects, Present Technology, and Future Problems, W.J. Anderson, ed., ASME, 1980, pp. 111-159.

[61] P.G. Morton, Measurement of the Dynamic Characteristics of a Large Sleeve Bearing, J Lubric Technol 93 (1971), 143-150.

[62] P.G. Morton, Dynamic characteristics of bearings: Measurement under Operating Conditions, GEC J Sci Technol 42 (1975), $37-47$.

[63] R. Brechting, R.J. Prior, R.D. Flack and L.E. Barrett, Load direction effects on measured static and dynamic operating characteristics of tilting pad journal bearings, in: Proc. 6th International Conference on Rotor Dynamics, E.J. Hahn and R.B. Randall, eds, IFToMM, Sydney, Australia, 2002, pp. 659-665.

[64] R. Nicoletti and I.F. Santos, Frequency Response Analysis of an Actively Lubricated Rotor/Tilting-Pad Bearing System, J Eng for Gas Turbines and Power 127 (2005), 638-645.

[65] R. Nordmann and K. Schöllhorn, Identification of Stiffness and Damping Coefficients of Journal Bearings by Means of the Impact Method, in: Proc. Vibrations in Rotating Machinery: $2^{\text {nd }}$ International Conference, IMechE, Cambridge, UK, 1980, $231-238$.

[66] R. Tiwari, A.W. Lees and M.I. Friswell, Identification of Dynamic Bearing Parameters: A Review, Shock and Vibrat Digest 36 (2004), 99-124. 
[67] R. Tiwari, A.W. Lees and M.I. Friswell, Identification of Speed-Dependent Bearing Parameters, J Sound Vibrat 254 (2002), $967-986$.

[68] R.D. Flack, G.J. Kostzrewsky and D.V. Taylor, A Hydrodynamic journal bearing test rig with dynamic measurement capabilities, Tribol Trans 36 (1993), 497-512.

[69] R.D. Flack, K.D. Wygant and L.E. Barrett, Measured Dynamic Performance of a Tilting Pad Journal Bearing over a Range of Forcing Frequencies, in: Proc. $7^{\text {th }}$ International Conference on Rotor Dynamics, Vienna University of Technology, Vienna, Austria, 2006.

[70] S. Hisa, T. Matsuura and T. Someya, Experiments on the Dynamic Characteristics of Large Scale Journal Bearings, in: Proc. 2 ${ }^{\mathrm{d}}$ International Conference on Vibrations in Rotating Machinery, IMechE, Cambridge, UK, 1980, 223-230.

[71] S. Xu, Experimental Investigation of Hybrid Bearings, Tribol Trans 37 (1994), 285-292.

[72] S. Yanabe, S. Yamashita and B. Weyh, A Method for Measuring Oil Film Coefficients of Journal Bearings, in: Proc. 3rd International Conference on Rotordynamics, M. Lalanne and R. Henry, eds, IFToMM, Lyon, France, 1990, pp. 85-89.

[73] S.H. Chan and M.F. White, Experimental Determination of Dynamic Characteristics of a Full Size Gas Turbine Tilting-Pad Journal Bearing by an Impact Test Method, in: Modal analysis, modeling, diagnostics, and control: analytical and experimental: Proc. ASME 1 ${ }^{\mathrm{h}} \mathrm{h}$ Biennial Conference on Mechanical Vibration and Noise, T.C. Huang, ed., Miami, FL, 1991, pp. 291-298.

[74] S.J. Kline and F.A. McClinktock, Describing Uncertainties in Single-sample Experiments, Mechanical Engineering: J of the ASME 75 (1953), 3-8.

[75] S.X. Zhao, H. Zhou, G. Meng and J. Zhu, Experimental identification of linear oil-film coefficients using least-mean-square method in time domain, J Sound Vibra 287 (2005), 809-825.

[76] T. Someya, ed., Journal Bearing Data-Book, Springer-Verlag, Berlin, 1989.

[77] V.N. Constantinescu, On Turbulent Lubrication, Proc IMechE 173 (1959), 881-900d.

[78] W. Dmochowski, Dynamic Properties of Tilting-Pad Journal Bearings: Experimental and Theoretical Investigation of Frequency Effects due to Pivot Flexibility, J Eng for Gas Turbines and Power 129 (2007), 865-869.

[79] W. Dmochowski and K. Brockwell, Dynamic Testing of the Tilting Pad Journal Bearing, Tribol Trans 38 (1995), $261-268$.

[80] W. Kang and D. Jin, The application of the model reference adaptive system in the identification of dynamic characteristics of oil-film bearings, in: Proc. Vibrations in Rotating Machinery: $4^{\text {th }}$ International Conference, N.K. Gakkai, ed., IMechE, Edinburgh, UK, 1988, pp. 241-246.

[81] Y.Y. Zhang, Y.B. Xie and D.M. Qiu, Identification of Linearized Oil-Film Coefficients in a Flexible Rotor-Bearing System, Part I: Model and Simulation, J Sound Vibra 152 (1992), 531-547.

[82] Y.Y. Zhang, Y.B. Xie and D.M. Qiu, Identification of Linearized Oil-Film Coefficients in a Flexible Rotor-Bearing System, Part II: Experiment, J Sound Vibra 152 (1992), 549-559.

[83] Z.L. Qiu and A.K. Tieu, Experimental Study of Freely Alignable Journal Bearings - Part 2: Dynamic Characteristics, J Tribol 118 (1996), 503-508.

[84] Z.L. Qiu and A.K. Tieu, Identification of sixteen force coefficients of two journal bearings from impulse responses, Wear 212 (1997), 206-212. 

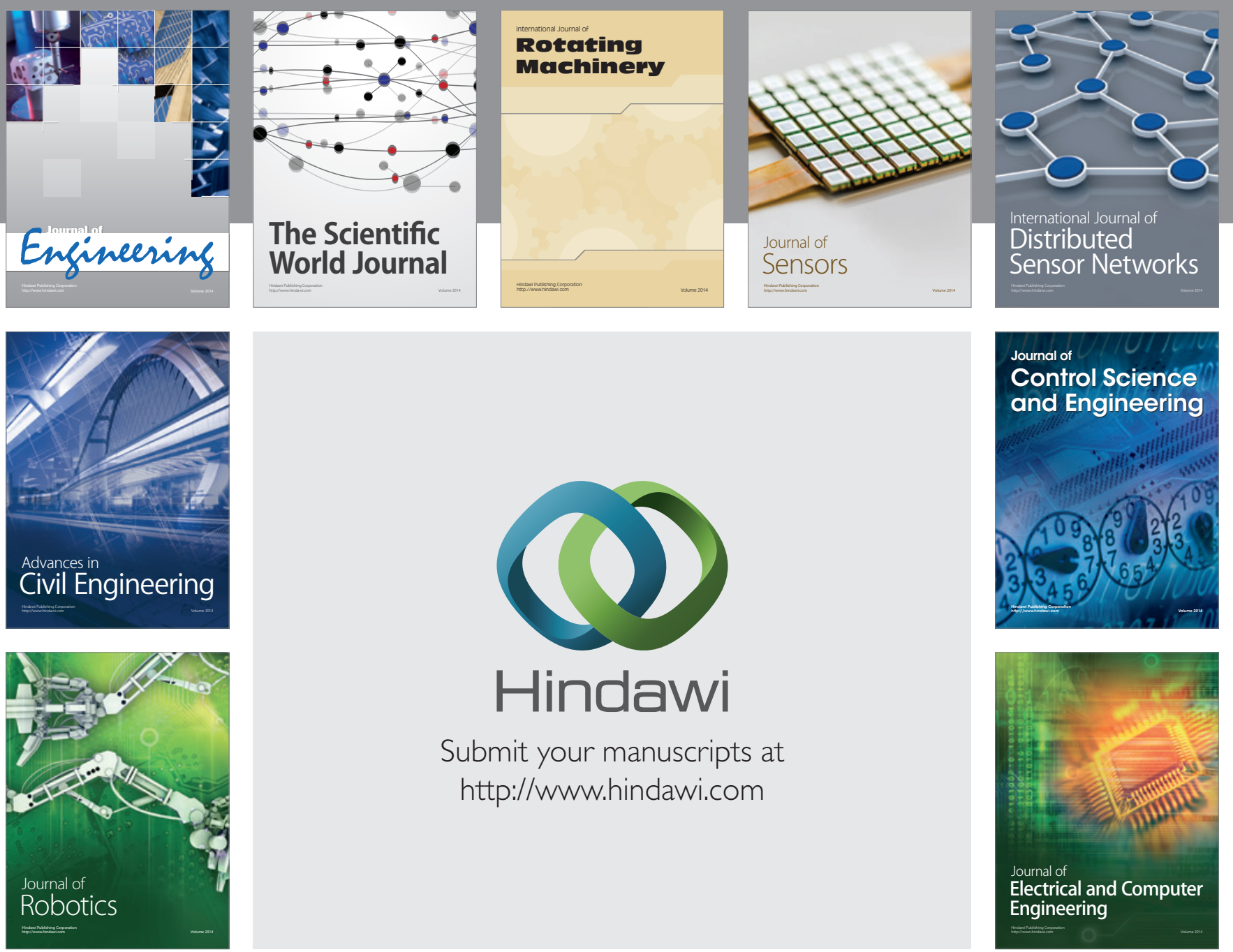

Submit your manuscripts at

http://www.hindawi.com
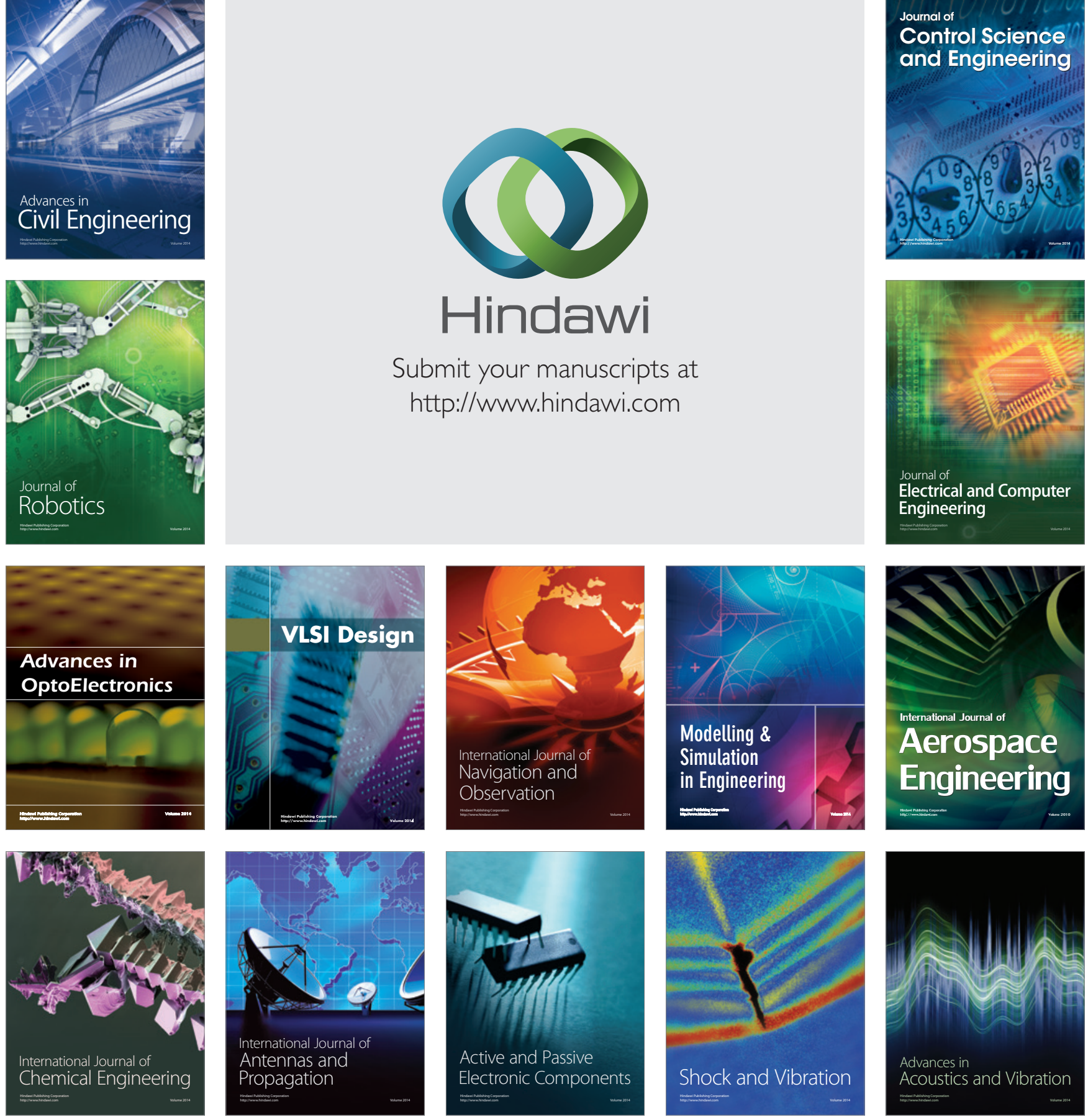